\title{
The place of Referent Tracking in Biomedical Informatics
}

\author{
Werner Ceusters
}

Departments of Biomedical Informatics and Psychiatry

Jacobs School of Medicine and Biomedical Sciences

University at Buffalo

77 Goodell street

Buffalo, NY - 14203

wceusters@gmail.com

Forthcoming in 'Terminology, Ontology and their Implementations', Springer Book 87020228, Springer Nature Switzerland AG

\begin{abstract}
Terminological systems, including coding and classification systems, are used in electronic medical record systems to facilitate the interpretation of structured data by providing terms and codes with a relatively precise meaning. When a clinician selects a term or code from such system and enters it in the medical record of a patient, then, from an ontological perspective and as a consequence of how terminological systems are currently integrated in electronic medical record systems, an assertion has been made to the effect that the patient exhibits, or exhibited, some phenomenon of type T. It is however left unspecified which phenomenon in particular is of the designated type $\mathrm{T}$. In other words: such records contain explicit references, i.e. the terms or codes, but the referents of these references are not explicitly identified! Because referents can be referenced in many different ways, types used as references can be about many referents, and referents may change so they become of a different type, data analytics application which rely on types only are prone to drawing erroneous conclusions. Referent Tracking is a methodology for data management which allows assertions only to be made with explicit reference to the referents they are about. This chapter offers an introduction to the principles upon which the methodology rests and how these principles can be applied to improve the quality of the problem list in medical records.
\end{abstract}




\section{$1 \quad$ Introduction}

The large majority of assertions in electronic medical records (EMR), whether in natural language or in structured form, are primarily descriptive: they are intended to provide a faithful account of facts relevant to a patient's medical history. That includes not only descriptions of what has been observed (signs and symptoms), hypothesized or concluded (diagnoses), or provided (a topical treatment, an injection of some sort), but also what has been ordered (lab tests, radiographies, etc.) or recommended (to take certain drugs in precise quantities at particular times). Assertions about recommendations and orders as registered in the EMR, in addition to being descriptive about the fact that they have been issued, are also prescriptive: with them comes an expectation that they will be carried out. Once that has happened, it may lead to further factual descriptions in the form of lab results and an updated medication list obtained through, for example, medication reconciliation.

For such descriptive statements to be useful, even if they contain faithful representations of a patient's medical history, they need to be understandable to their intended audience, and not only to their original author. This audience does not only include clinicians and other healthcare workers, but also software applications performing automated decision support and predictive analytics. While the former can deal with assertions in natural language, the latter still require formally structured assertions despite some advances in natural language understanding.

This chapter focusses on a specific design flaw in information systems and data repositories in general and EMR systems in particular which renders data less understandable, and thus less useful, than what one would expect, especially for data analytic purposes. This flaw is that the majority of the structured assertions EMR systems contain lack an explicit reference to that what these assertions are precisely about. Part of the problem is the false belief that EMR data, because they are expressed in terms of codes that are given a precise and standardized meaning, are therefore themselves precise and unambiguous. This is of course absurd. One can, for example, safely assume that within the confines of a hospital the word 'doctor' has a very precise and generally well-understood meaning. But what are the chances that a nurse who wants to draw the attention of a specific doctor walking by her office in the company of a number of colleagues would make the head turn only of the doctor she wanted to talk to, simply by yelling 'doctor!'? What is required to remove this sort of ambiguity, is clear identification - the sort of clear identification that is provided in normal life by, for example, proper names and social security numbers. It is precisely clear identification and how to use clear identification in structured biomedical data in combination with terms from terminologies and ontologies that is at the heart of Referent Tracking (RT). RT offers a methodology for making assertions following specific principles (sections 2). These principles are themselves grounded in realism-based ontology (section 3) and form the basis for determining what it is on the side of reality that can and should be uniquely identified under a given ontological perspective (section 4). RT representations consist of 
predicates called 'RT-tuples', each one of which is derived from a tuple type which has a precisely defined abstract syntax and semantics and is to be used for a precisely defined declarative purpose leaving almost no room for unintended ambiguity (section 5). That this approach has value is exemplified by means of a case study of a problem list from a medical record (section 6) and an analysis of how this problem list can be rendered more useful for data analytics under a number of scenarios at different levels of granularity (section 7) with the help of RT. The chapter concludes with suggestions for further reading (section 8).

\section{Predication}

Whatever the audience of an assertion in an EMR, there are at least two requirements for it to be useful: it must be clear (1) what precisely is attributed - i.e. what features, characteristics, properties, etc. are ascribed to some entity relevant to the patient's health - and (2) about what it is attributed - i.e. to which entity the features, characteristics, properties, etc. are ascribed. The part of a descriptive statement that ascribes features to some entity will from here on be referred to by the term 'predicate', with the terms 'predication' or 'predicating' denoting the process of ascribing characteristics to something. The entity about which something is predicated, will be referred to as the 'referent'. A descriptive statement of this sort will be referred to as an 'ascription'.

\subsection{Predication and Terminology}

Currently, EMR systems do not come with built-in natural language understanding capabilities and those which do, offer not more than some form of phrase-based semantic indexing without deep semantics. To render EMR data maximally useful for analytic purposes, predication capabilities in EMR systems therefore rest mainly on structured data registration forms which emphasize the 'what'-part of predicates, and limit input - leaving aside here numeric data - to what is offered by means of terminologies. Biomedical terminologies are designed primarily to standardize the semantic content of a predicate independent from syntactic form. More precisely, terminologies help authors of descriptive assertions to avoid potential ambiguity in what is predicated, and, as a consequence, they help the intended audience of such descriptions to understand them in the intended way insofar as they are familiar with the specific biomedical terminologies used. This is because biomedical terminologies organize terms that can be predicated on the basis of what are called 'concepts' [1]. Each concept is represented by a preferred term and, when present, also a concept identifier and a number of synonyms. The SNOMED CT identifier (SCTID) '289900009', for example, identifies a concept that is linked to four English terms: 
'Period pain', 'Period pain present', 'Painful menstruation', and 'Period pain present (finding)'. 'Period pain present' is thereby qualified as being the preferred term, 'Period pain present (finding)' as the fully specified name, and the two other terms as synonyms.

But clearly, when a clinician selects the SNOMED CT term 'Period pain' for addition to some patient's problem list in an EMR, the referent of the predication is not the concept to which the selected term is linked, but rather some entity on the side of the patient. The question now is: which entity precisely? What is the intended referent? There are indeed several closely related, but nevertheless very distinct entities that would qualify for being the referent of the predicate. Is it the patient herself? Is it the complaint that she is expressing? If not the complaint, is it then that what the complaint itself is a description of, which can be either the particular type of pain the patient suffers from whenever she menstruates, or, for example, the specific occurrence of this type of pain during her most recent menstruation? Conceptbased biomedical terminologies take care of what can be predicated in a standardized and relatively unambiguous way, at least in the sense that the terms they contain give a good idea of what happened somewhere on the side of the patient. But they come not equipped with a mechanism to specify the concrete referent of a predicate [2]. Unfortunately, they are often even misleading; indeed, the variety of terms for the concept SCTID-289900009 makes one wonder whether the pain is the referent, the menstruation, or perhaps, as suggested by the fully specified name, the finding - the fact of observing - the pain!

In general, when in descriptive statement $d$ the term $t$ from a terminology is used to predicate $p$ about some entity $e$ on the side of the patient $-e$ being, for example the patient's blood pressure, his heart, or his pain - then $t$ itself does not convey formally that $e$ is denoted. This is what may be called 'referent ambiguity'. There are some exceptions though: the referent of the SNOMED CT term 'United States of America (geographic location)' (SCTID:223688001) is quite obvious, as there is only one United States of America. But imagine that during a consultation a clinician, assisted by a nurse, says to his patient: 'this is a malignant nevus' thereby pointing to the patient's left cheek. In this sentence, 'malignant nevus' is the term used in the predicate 'is a malignant nevus' which is ascribed to the referent of 'this'. Clearly, the referent of 'this' is not the cheek, but more likely a mole on the patient's cheek. But what if there are three moles? In that case, the clinician should not have been pointing merely to the cheek, but to that one mole out of the three which he diagnosed as being malignant. In this case, it is the combination of the pointing and the use of the demonstrative 'this' that allows the addressee of the phrase 'this is a malignant nevus' to uniquely identify what the utterer of the phrase is precisely talking about. Without the pointing and the use of 'this', any mole on the patient might qualify for being the referent. For the assisting nurse, the term 'malignant nevus' merely provides support for her identifying - though it does not identify - the exact referent as she probably understands what counts as a 'nevus'. The patient, when unfamiliar with that term, is provided with no support for his understanding of what the referent is. But he might have learned something, i.e. that that what he knows as 'mole' qualifies also as 'nevus'. 


\subsection{The need for unique identification}

Interestingly, a similar mechanism - equivalent to pointing or to the use of demonstratives - for unique identification of the referents of predicates in an EMR is generally lacking [3]. Certain unique identifiers as currently used in some EMR systems, whether or not visible as such in the user interface, would qualify as being an analogue for pointing, but they are only used for certain sorts of referents such as patients and providers and also organizations. However, there are currently, to the best of our knowledge, no EMRs that use unique identifiers for, for example, the individual disorders patients are suffering from or for the individual body parts in which such disorders are present. There might be unique identifiers for the type of disorder, or the type of body part, for instance when SNOMED CT is used. These identifiers describe an individual patient's individual disorder resp. body part, but they do not uniquely identify them. After all, if each of two distinct patients suffer from a disorder of exactly the same type, the same SNOMED CT code would be used, yet, the two disorders are obviously distinct: one disorder is in one patient, and the other in another patient. Furthermore, a single patient might suffer from the same type of disorder more than once, at different times and even at the same time.

At the other hand, unique identifiers in the form of primary keys are extensively used behind the scenes in the back-ends of EMR systems. They can be found in tables in which data related to problem lists, treatments and appointments are stored. It is, however, much less clear what it is precisely that is uniquely identified by these primary keys: is it the data entry itself - a piece of information - or is it the referent of that piece of information: some phenomenon on the side of the patient which requires medical attention? If it is only record entries which are uniquely identified, then there is no way to distinguish whether two distinct entries, for instance 'Melanocytic nevus of neck (disorder)' entered in January 2018 and 'Malignant melanoma of neck (disorder)' entered in 2020 are about the very same lesion the patient is suffering from or about two distinct lesions. In the former case, the two entries would indicate that the benign nevus turned malignant; in the latter, the two nevi are diagnosed as being unrelated to each other. As the example demonstrates, this sort of ambiguity in EMRs exists not because of the use of ambiguous terms but because of a double disconnect between, on the one hand: (1) the ways in which EMR systems structure the information they contain, and the ways in which entities in reality are structured, and on the other hand: (2) the extent to which changes in the EMR's information correspond to changes in reality.

\subsection{Predication Principles of Referent Tracking}

It is to prevent and, so far as is possible, to resolve these sorts of ambiguities in EMRs that Referent Tracking (RT) was developed [4, 5]. RT is a regimented methodology for rendering data in data repositories in general and EMRs in particular in 
a way that is much more explicit than through mainstream approaches. A number of Referent Tracking Principles (RTP) have been specified below for those who wish to use the methodology appropriately. However, depending on the purpose for which a specific Referent Tracking System (RTS) is designed, some of these principles might be relaxed. An example is that ascriptions must be designed following RT-specific syntactic-semantic conventions and stored in a Referent Tracking Database (RTDB). The RTDB may then either form part of the back-end database of an EMR system, or be separately maintained in a RTS in which case there must be semantic interoperability between the EMR system and the RTS. Some principles need to be adhered to by the EMR system or the RTS, for instance RT-specific principles for keeping the RTDB internally consistent and also with what is predicated in other parts of the EMR [6-9]. Other principles are to be followed by users entering information in the EMR. These principles are at the highest level:

RTP1. A predicate shall be made about one or more referents if and only if these referents are uniquely identified in the RTS (RTP1.1) and the author of the predicate holds at least two beliefs: (1) that the referents are real, were real or very likely will become real (RTP1.2) and (2) that what is predicated about these referents - within the specified time-frame when time-indexing is required - is either true, or more likely true than false. In the latter case, the strength of the belief in the correctness of the predicate needs to be expressed as well (RTP1.3). This holds for positive ascriptions as well as negative ascriptions, i.e. ascriptions positing that something is not the case [10].

RTP2. The RTS must keep track of the provenance of assertions, thus by whom and when predicates were asserted, confirmed, contradicted, corrected or rejected in the EMR (RTP2.1) and by whom and when such changes were registered in the RTS (RTP2.2).

RTP3. The RTS must use the ascriptions to construct a representation of reality from the perspective of one or more clearly specified ontologies which are sufficiently expressive and comprehensive to accommodate all types of ascriptions in the EMR (RTP3.1). This representation must be updated whenever the pool of ascriptions in the EMR is updated (RTP3.2). Ascriptions which when taken together do not allow a representation to be constructed which is free from contradictions and inconsistencies need to be identified (RTP3.3) and appropriate reconciliation procedures invoked (RTP3.4).

These principles form a solid basis to identify and remediate shortcomings not only in how users of data repositories in general and EMRs in particular register their observations and findings, but also in the design of enabling technologies used for that purpose such as data entry screens and the information models behind them, as well as terminologies, classification systems and ontologies [3, 6, 11-16]. It can even be argued that it is only through the application of these very principles that collaboration between domain experts and ontologists can lead to high quality biomedical ontologies $[17,18]$. 


\section{Ontological Foundations of Referent Tracking}

Using RT for predications in a way that satisfies the principles described in section 2.3 requires adherence to a view on reality based on Realism, more specifically Metaphysical Realism, i.e. the doctrine according to which universals exist in reality, and Scientific Realism, i.e. the doctrine according to which scientific theories are (broadly) true of reality. Both doctrines form also the basis of Ontological Realism [19] which in turn has been used to lay the foundations - of which unfortunately quite a bit is lost in implementations - of the upper ontology 'Basic Formal Ontology' (BFO) [20] which is now approved as standard ISO/IEC 21838-2, and several biomedical ontologies of which the most relevant ones for the application of RT in biomedicine are the Information Artifact Ontology (IAO) [21], the Relation Ontology (RO) [22], the Ontology for General Medical Science (OGMS) [23] and the Ontology for Biomedical Investigations (OBI) [24].

\subsection{RT adherence to $\mathrm{BFO}$}

It is not required to use $\mathrm{BFO}$ and $\mathrm{BFO}$-compatible ontologies for RT; any ontology based on metaphysical and scientific realism and which is based on a wellfounded theory of what exists and is the case in reality, might do. There are however several advantages in using BFO. This is because both RT and BFO are designed following the same underlying principles the most central ones being that representations should (1) be faithful to reality and (2) have an abstract structure which mimics the structure of reality. These two principles should not be taken to imply that BFO and RTDBs are indeed faithful. But at least they mean (1) that their respective authors believe they are faithful, and (2) that whenever a representation fails to be faithful, it needs to be corrected. The benefits of BFO and RT adhering to these principles can be observed both in the creation of new representations as in the quality control of existing ones. BFO, when used appropriately, provides solid foundations for the development of other realism-based ontologies. RT similarly provides solid foundations for the construction of databases that intend to follow the same central principles. Where BFO has been shown to be instrumental in detecting inconsistencies and ambiguities in formal terminologies such as SNOMED CT [2530] and classifications such as the International Classification of Headache Disorders [31, 32] and the Research Diagnostic Criteria Matrix for mental disorders [33], RT has been used to find inconsistencies and ambiguities not only in EMRs [6, 11, 12] but also in common data models designed to pool data from different EMR systems [13-16], as well as in areas of biomedical research in which the terminology is not yet fully developed such as bruxism [34] and medically unexplained syndromes [35].

When BFO and the realism-based ontologies that use BFO as top ontology are used for RT, then it is also not a requirement to use them completely 'as is'. Several 
alternative views and perspectives can be integrated in an RTDB as long as the principles of Ontological Realism and RT are adhered to. One alternative, for example, is to extend BFO with additional defined classes and relations as long as they do not conflict with any of the existing axioms, an approach that is possible since $\mathrm{BFO}$ does not claim exhaustiveness of the entities it represents. The new ISO/IEC 21838-2 standard provides detailed rules governing how to build BFO-conformant ontologies. An example of such extension would be to allow RT-predicates to be about 'temporally qualified continuants' [36]. Another alternative is to include 'object clusters' and 'object groups' as defined classes under BFO's 'object aggregate' [37], and to include 'portion of matter' as a defined class under BFO's 'material entity' [38]. These proposals allow a more accurate representation of, for example, how organs and tissues are composed out of cells. This will also allow the definition of a BFO-conformant means to refer, for example, to portions of liquid, portions of gas, portions of plasma, and so forth.

Also allowed in RT is to move outside what currently can be represented through $\mathrm{BFO}$ while staying within what Ontological Realism considers to be real. Configurations, from certain perspectives comparable to, but nevertheless different from states of affairs and facts [39], would be an example. A configuration is a portion of reality (PoR) - i.e. a part of reality 'sliced' or 'carved out' in any way - which consists of a particular and at least one other entity related thereto in a specific way, for example that Michael J Fox (a particular) suffers from his Parkinson's disease (another particular) and that his Parkinson's disease is an instance of disease (a universal). 'Representation', 'ascription' and 'predicate' are throughout this chapter thus not used in the narrow sense such that 'representation' and 'ascription' would only apply to entities in BFO's sense and 'predicate' to the 'the standard predicates of first-order logic fantologically conceived' [40]. Rather they are used in the sense that all representations, ascriptions and predicates are about something real or about being the case - whether veridical or not - and can be expressed in any suitable formal or natural language [21].

Another example of what is allowed to be included in a RTDB are RT-ascriptions that relate entities to concepts that figure as nodes in concept-based terminologies such as SNOMED CT. It would of course not be allowed to use the instance relation as defined in BFO in such a case. Rather one would formally define within the RTS - or if working on a larger scale and for global use - what could be called an annotation ontology comprising a number of relations each one of which expresses the specific semantic and pragmatic conditions that must be fulfilled for that relation to be used to annotate some PoR with some concept code. It is of utmost importance that it is fully specified which concept code from which version of which representational resource is intended as for many such resources the meanings of codes and concepts change [41-43]. 


\subsection{Ontological distinctions essential for RT}

An ontology suitable for use in RT must make a number of ontological distinctions. The first forms the foundation for Referent Tracking Principle RTP1: the distinction between representations such as ascriptions and what the predicates of these acscriptions are about, i.e. their referents. This distinction is not explicitly made in BFO itself because representations are in the realm of the IAO [21]. RT, however, requires explicit referencing not only of entities to which something is ascribed in the RTDB but also of the ascriptions themselves, and this because of the requirement that RT systems must track the evolution of their RTDB (RTP3). A similar approach has been suggested for BFO updates but this has not been implemented thus far [44]. RT distinguishes further between representations that are internal, i.e. residing in the RTDB, and representations that are external. A further distinction amongst external representations separates beliefs, i.e. cognitive representations of reality on the part of, for example, researchers from concretizations of these cognitive representations in (for example textual or graphical) representational artifacts [45]. Representations can of course have representations as parts. A medical record is a representation of a patient's medical history. If it contains a blood lab report, then that lab report is a representation of part of what was the case as concerns the blood of that patient at the time the blood was examined. If on that lab report there is a part on which is printed 'glucose: $75 \mathrm{mg} / \mathrm{dl}$ ', then that part is a representation of the blood glucose level in that blood. The word 'glucose', however, being in this case a representation for the glucose in that patient's blood sample, cannot be further subdivided in further representations. A representation that has no further representations as parts is a representational unit (RU). That a RU has no further representational parts does not entail that what is denoted by it does not have further parts. Of course, what counts as a RU depends on the sort of representational machinery used. The word 'glucose' is a RU that does have parts, i.e. each individual letter, but these letters are not RUs. The symbol ' $\mathrm{C}_{6} \mathrm{H}_{12} \mathrm{O}_{6}$ ' is a $\mathrm{RU}$ which has parts ' $\mathrm{C}_{6}$ ', ' $\mathrm{H}_{12}$ ', ' $\mathrm{O}_{6}$ ' which are composed of the further parts ' $\mathrm{C}$ ', ' $\mathrm{H}$ ', 'O', ' 12 ' and twice ' 6 ', and all these parts are RUs as each one of them denotes some PoR. Up to a certain level of granularity, ' $\mathrm{C}_{6} \mathrm{H}_{12} \mathrm{O}_{6}$ ' satisfies the principle that representations should 'mimic the structure of reality'. The symbol mimics what a glucose molecule is made of, without, however, mimicking its three-dimensional structure.

A second required distinction to be recognized in an ontology suited for RT is the one between PoRs which are specific (non-repeatable) and PoRs which are generic (repeatable). For BFO, this is the distinction between particulars, i.e. entities each one of which occupies a unique spatiotemporal location and instantiates at least one universal [20, p182], and universals, each one of which is - or ever has been instantiated by at least one particular and depends on that particular for its existence $[20, \mathrm{p} 186]$ respectively. An exception in BFO are generically dependent continuants (quality patterns, for instance DNA sequences) which are repeatable in the sense that they can be copied. but they are not universals. 
RT can accept other PoRs as being non-repeatable if so recognized by the ontology used as basis. An example would be facts as the truthmakers for true ascriptions, e.g. the fact that this chapter is written in English, the fact that Werner Ceusters is the name of the author of this chapter, etc.

The third distinction to be made is between continuants and occurrents. This distinction determines both in BFO and RT the requirement for temporal indexing of assertions about the former. In BFO, it is responsible for the different interpretation that must be given to assertions of the sort ' $U 1$ isa U2'. When U1 and U2 are occurrent universals, the interpretation is that all instances of $U 1$ are instances of U2 independently of any reference to time. In the case of continuant universals, the interpretation is that all instances of $\mathrm{U} 1$ are instances of $\mathrm{U} 2$ at the time they are instances of U1. This difference is unfortunately lost when BFO is represented naively in OWL by substituting BFO's isa relation for OWL's subClass Of relation. Explicit time-indexing is in BFO also used for any axiom that involves a continuant as well as for the definition of instance-level relations involving a continuant. For $\mathrm{RT}$, the distinction translates in a similar requirement that whenever an RTascription contains a RU denoting a particular - or in general a non-repeatable PoR with continuant-like behavior - the ascription must contain an explicit reference to the time during or at which the relation expressed in the ascription holds. A useful resource for ontology-based temporal-indexing is ISO-standard 12381:2019 which resulted from earlier work carried out under the realm of the European Centre for Standardization $[46,47]$.

Finally, the distinction between dependent and independent entities is implemented at the highest levels in BFO: (1) all universals ontologically depend on particulars, (2) the immediate subtypes of continuant are independent continuant, specifically dependent continuant and generically dependent continuant, and (3) the immediate subtypes of occurrent are temporal regions and spatiotemporal regions as independent entities and processes and process boundaries as dependent entities. For RT, the distinction between ontologically dependent and ontologically independent PoRs is extremely useful in deciding what particulars need to be represented in a RTDB. As a general rule, whenever a dependent entity is represented, one should also represent all entities upon which it ontologically depends [6].

\section{$4 \quad$ Unique Identification in Referent Tracking}

An essential notion in RT is unique identification. Unique identification rests on a view which acknowledges the existence of PoRs that are numerically unique in the sense that they carry identity according to one or other standard. Identical twins are (to a certain degree) qualitatively identical to each other but not numerically identical. It is numerical identity that is the topic of unique identification. The goal of RT is a situation in which numerically unique PoRs are referenced by identifiers that are singularly and globally unique, either within a single RTS or within a network of RTSs that exchange information. An identifier is globally unique when it 
denotes precisely one PoR. An identifier is singularly unique when the PoR it denotes is not denoted by another identifier. Unfortunately, this ideal is not always attainable, influencing factors being the ontological view under which an RTS is chosen to function, and the ambiguity of predicates as for instance found in EMR data that are used as a basis for populating an RTS. As a consequence, the identifier system of an RTS must be set up in such a way as to accommodate for uncertainty with respect to uniqueness.

\subsection{Which PoRs are numerically unique?}

Which PoRs are objectively numerically unique depends on the ontological stance one supports and on the perspective from which reality is observed. When an RTDB is used exclusively in conjunction with BFO-based ontologies, then the only PoRs that qualify for unique identification are (1) particulars, (2) the particularto-particular and particular-to-type relations defined in BFO, and (3) the types figuring as nodes in BFO's taxonomy. Although BFO's taxonomy contains only nodes denoting types, the taxonomic relations, as well as any other type-type associative relation, should not be interpreted as statements about types; rather, they are statements about how any instance of one type stands in the specified relation to some instance of the other type. Outside BFO, but inside an RTS using an ontology that includes universals in its domain of discourse, it is perfectly possible to make assertions about a uniquely identified type [40]. This is possible because both BFO and $\mathrm{RT}$ subscribe to 'perspectivalism' i.e. 'the view according to which reality is too complex and too variegated to be embraced within a single scientific theory' [20, p183]. The standard BFO-perspective is that particulars can be classified on the basis of the universals they instantiate, the latter providing the backbone taxonomy of BFO or of the relevant BFO-compatible domain ontology, and the relations they enjoy with other particulars, allowing, for instance, for a further categorization in defined classes such as 'citizen of Buffalo NY' [45]. Under another perspective, universals can themselves be categorized, for example according to the number of particulars that instantiate them, or whether they have a taxonomy of subtypes that can be mapped on a continuous scale such as temperatures and speeds, in contrast to, for example, what is the case for object-universals such as 'thermometer' or 'cell'. A classification of this sort has been proposed for clarifying terminological problems concerning the definition of bruxism [34] and for a classification of mental disorders satisfying both the dimensional and categorical paradigms [48]. Both of these examples illustrate the danger in unwittingly running together two forms of speech governed by different logical rules.

Collections of particulars are also unique but require special attention, not least because in many cases what one would denote as 'the collection' is a different particular than the totality of the entities that make up that collection. A heap of stones, for example, can cease to exist without any of the stones that make up the heap ceasing to exist. On the other hand, a surgeon's team does not cease to exist when 
a member of the team leaves, unless the person who leaves is the last member of the team and the ontology used requires of a team that it consists of at least one member.

Extremely relevant for healthcare is that representations also be unique. An entry, for example a diagnosis in a patient's problem list in some patient's medical record, is a representation. In a typical case, a diagnosis is about some disease in a patient; diseases are in patients, diagnoses are in medical records and in the brains of clinicians. A patient can have two diagnoses even though he is suffering only from one particular disease. These two diagnoses might be exactly similar in that they assert that that patient's disease is an instance of disease universal $D_{l}$. Or they might be distinct, as when one diagnosis represents the disease as being of type $D_{1}$ and the other one as being of type $D_{2}$. When distinct, both might be correct, for example because one is less precise than the other one, e.g. 'liver tumor' versus 'malignant liver tumor'. Separating representations from what they are - or might be - about is not only crucial in case of litigation, but also when representations are used to develop decision support systems on the basis of, for example, deep learning.

\subsection{Using identifiers in RT}

RT requires each uniquely identifiable PoR about which information is to be stored in an RTDB to be represented by means of a Referent Unique Identifier (RUI). For PoRs which are particulars, the name Instance Unique Identifier (IUI) is used as well because it is only particulars that can instantiate a universal. Whenever, for example, a clinician predicates in an EMR something about a PoR to which nothing has been ascribed before, then the RTS of the EMR system must generate a RUI for that PoR. There is no specific format requirement for RUIs; being unique is the only requirement.

Within the RTS, RUIs are to be treated as proxies for the PoRs to which they are assigned; each RUI is comparable to spectacles through which one can see only the PoR to which it has been assigned. Extreme care must therefore be taken to provide an accurate description of what is exactly intended to be denoted by the new RUI, thereby considering that terminology might be very confusing when time-indexing is ignored. Without accurate description, it is difficult to assess whether a PoR one wants to predicate about has already been assigned a RUI in the RTS. If the RTS has already information about that PoR, the corresponding RUI should be retrieved and be used in the creation of new predicates about that PoR.

A RUI is a representation that denotes, i.e. identifies - or is intended to identify - a PoR in a globally unique way within the confines of an RTS or RTS network. For most PoRs, RUIs should ideally also be singularly unique. For some entities, even ones that are numerically unique, this property cannot always be guaranteed at the time the identifier is to be assigned. This happens often with identifiers for 
temporal regions because it is in most cases very hard, if not impossible, to determine whether two numerically distinct entities are related to two other numerically distinct entities within exactly the same temporal region. When, for example, two babies are conceived at exactly the same time, then at that very same time their lives came into being. For each of these two babies, there is a temporal region during which each own participates in its own life. If each of these two configurations is to be represented in an RTS, then two distinct representations are to be created, one for each baby, each one of which must be time-indexed because babies are continuants. But whether there is only one temporal region towards which the predicates must be indexed, or two distinct ones, cannot be determined as long as both lives continue. If they should terminate at the same time, then there was only one temporal region involved, otherwise two, and this already from the very beginning with the conception of the babies. This is one example of what under the view of Ontological Realism is meant by the claim that reality is objectively the way it is and that it is partly accessible to us. Ontologically, it is not a possibility that it is one or two temporal regions that are involved but a fact that it is one or the other. But the part of reality that is accessible to us prior to the termination of at least one life is not sufficient to determine which of the two is the case. As another example, it is in many cases also impossible to determine what the temporal boundaries of a temporal region are. When precisely did the diabetes start of a patient with type 2 diabetes? Once again, ontologically there was a beginning, be it epistemologically undeterminable.

Singularly unique identification might also be problematic in other situations as well. If, for example, the SNOMED CT code '283734005 | Dog bite - wound (disorder) |', appears in an EMR system on the problem list of several patients, there is in the absence of further information no way of being sure whether it was in each case a different dog. If several patients are documented as being the victim of '242406007 | Fall due to impact against supermarket shopping cart (event) |', there is similarly not enough information to determine whether these falls happened in the same or in a different supermarket, with the same or with a different cart. Knowing these details might not have an impact on the course of action to be taken in the care of the patients involved, but it might certainly influence decisions on public safety measures towards a specific dog or a specific supermarket.

For relations, universals and defined classes, some form of unique identifier is typically provided by ontologies either as a linguistic expression or as a code. There is however no guarantee that they are unique within the collection of ontologies selected to be used for an RT application. Moreover, for most terminologies and concept-based ontologies there is no guarantee that these identifiers will continue to denote exactly the same PoRs when an updated version is released. It is therefore strongly advised to ensure unique denotation of what is denoted in ontologies through appropriate additional mechanisms in the RTS. 


\section{$5 \quad$ RT Representations and their Management in an RTS}

RT representations consist of collections of tuples. A tuple is mathematically defined as a finite set of ordered elements. Tuples were introduced quite early in the history of knowledge representation in the form of simple (Entity, Attribute, Value) (EAV) tuples [49, 50]. EAV-tuples generalize the semantics of tables in relational databases (RDB). Each RDB-table consists of a variable number of rows, each row thereby representing some entity, and a fixed number of columns, each column corresponding to some attribute the entities possess. The cells contain the values for the attributes. An EAV-design can be thought of as consisting of only one table in which the columns of an RDB-table are converted to rows.

\begin{tabular}{|l|l|l|l|}
\hline Entity & Length & Weight & Price \\
\hline $\mathrm{E} 1$ & $5 \mathrm{ft}$ & $6 \mathrm{~b}$ & $\$ 23$ \\
\hline$\ldots$ & & & \\
\hline
\end{tabular}

\begin{tabular}{|l|l|l|}
\hline Entity & Attribute & Value \\
\hline E1 & Length & $5 \mathrm{ft}$ \\
\hline E1 & Weight & $6 \mathrm{lb}$ \\
\hline E1 & Price & $\$ 23$ \\
\hline$\ldots$ & & \\
\hline
\end{tabular}

Figure 1. Comparison of a relational table design with an EAV-design.

One could, for example, express that patient John Doe has Type 2 Diabetes by creating the EAV-tuple (John Doe, hasDisease, Type 2 Diabetes). If one were to apply the time-indexing requirement for continuants, then this EAV-tuple must be extended as, for example, in (John Doe, hasDisease, Type 2 Diabetes, since September 2007).

EAV-tuples and extensions thereof as n-tuples became popular because of their flexibility and intuitive semantics, a major drawback being, however, the lack of formal semantics. RT addresses this issue through the definition of a number of tuple types. Each tuple falls under precisely one tuple type, thereby being composed out of representational units each one of which denotes - i.e. intends, is directed towards [21] - some portion of reality. The referent of at least one RU in a RT tuple denotes a portion of reality which is uniquely identifiable and not repeatable. Of the referents that do carry identity, there is one which is the prime referent of the tuple. Referencing must always be done explicitly. RT does thus not allow - modulo some precisely defined exceptions - the use of 'shortcut' relations that relate, for example, a particular $p$ to a universal $U$ of which $p$ is not an instance thereby bypassing reference to an existing particular $q$ that is instance of $U$, as in 'John Doe exemplifies high blood pressure today' [40, Fig 4, p167]. The bypassed $q$, in this case, is John Doe's blood pressure which is today an instance of high blood pressure. It is for this reason that the extended EAV-tuple (John Doe, hasDisease, Type 2 Diabetes, since September 2007) used as example in the previous paragraph cannot be translated as such in one RT tuple. This is because 'Type 2 Diabetes' is therein used as universal and 'hasDisease' as a specialization of 'exemplifies'. Representing this in RT would 
require at least two extended EAV-tuples: (John Doe, hasDisease, \#1, since September 2007) and (\#1, instanceOf. Type 2 Diabetes, since September 2007).

The requirement for an RT-tuple to reference at least one uniquely identified non-repeatable PoR has as consequence that an RTDB does not contain any generic tuples such as (Mammal, isa, Vertebrate). These sorts of representations belong to the realm of ontologies. Despite not being part of an RTDB, they are used in an RTS to keep the RTDB faithful to reality. When various ontologies are used, possibly from distinct perspectives, then it might also be necessary to develop bridging axioms between terms [51]. These axioms, too, are not part of the RTDB, but are to be used in reasoners used by the RTS to keep the RTDB faithful.

RUs that do not represent non-repeatable PoRs are terms taken from - ideally realism-based ontologies. What can be predicated about PoRs in RT tuples is determined by constraints in these ontologies. The RUs themselves, as well as the tuples formed with them, figure as references, while what they are directed towards are the referents. Collections of tuples in which the same referent is represented are a means to track that referent, hence the name 'Referent Tracking' for the entire paradigm. RT requires each reference to be tracked as well and offers to that end principles which can be implemented in a Referent Tracking System without leading to an infinite regress [6-9]. The main goal of this is to identify and correct representational mistakes [52] or inconsistencies between references and referents brought about by various kinds of changes, reflecting for example: (1) changes in the underlying reality; (2) changes in our understanding; (3) reassessments of what is considered to be relevant for inclusion in a referent tracking system, or (4) encoding mistakes introduced during data entry [53].

\subsection{RT tuple types}

RT tuples come in a number of tuple-types. Tuple-types provide an abstract syntax for RT representations together with a direct referential semantics [9]. Examples are the tuple-type through which one can assert that some particular is an instance of some universal, or the tuple-type for asserting that some non-repeatable PoR stands in a certain relation to some other non-repeatable PoR, and so forth. These tuple-types were in version 1 originally defined to work exclusively with BFO and BFO-compatible ontologies [9]. Table 1 provides an updated perspective along the lines described in section 3.1 and allowing for an explicit representation of the principles described in section 2.3. Concrete examples of how these tuple types are applied is discussed in section 7 . 
Table 1. RT tuple types

\begin{tabular}{|c|c|}
\hline Tuple type & Format \\
\hline \multicolumn{2}{|l|}{ Description } \\
\hline A-tuple & $A \#<R U I_{a}, R U I_{p},{ }^{\prime} A{ }^{\prime} / R^{\prime}, '+S U ' / 6-S U^{\prime}, t>$ \\
\hline $\begin{array}{l}R U I_{a} \text { assign } \\
\text { uniquely ide } \\
\text { larly ('-SU') } \\
\text { sion } 1 .\end{array}$ & $\begin{array}{l}\text { (' } A \text { ') or reserved (' } R \text { ') at temporal instant } t \text { 'RUIp' to/for a } \\
\text { iable PoR either as singularly (' }+S U^{\prime} \text { ') or potentially non-singu- } \\
\text { que identifier. This tuple type extends the A-tuple type from ver- }\end{array}$ \\
\hline
\end{tabular}

D-tuple $\quad D \#<R U I_{d}, R U I_{T}, t, ' I ' E, R, S>$

$R U I_{d}$ registered at temporal instant $t$ tuple $R U I_{T}$, i.e. a tuple other than $D \#$ itself, either as an insertion ('I') in the RTS for reason $R$ [44], or as the correction of an error of type $E$ [54] in which case $S$ is a list of RUIs denoting the tuples, if any at all, that replace $R U I_{T}$. This tuple type is unchanged since the previous version.

\begin{tabular}{l}
\hline $\boldsymbol{F}$-tuple F\#<RUI, $\boldsymbol{t}_{\boldsymbol{a}}, \boldsymbol{R U} \boldsymbol{I}_{\boldsymbol{a}}, \boldsymbol{R U I} \boldsymbol{T}, \boldsymbol{C}>$ \\
$R U I_{d}$ asserts at temporal instant $t_{a}$ that his confidence in the faithfulness of tuple \\
$R U I_{T}$ as authored by $R U I_{a}$ is of level $C$. This tuple type did not exist in the previ- \\
ous version of RT.
\end{tabular}

NtoI-tuple NtoI\#< '+'/'-', $\boldsymbol{n t} \boldsymbol{j}, \boldsymbol{n}_{\boldsymbol{i}}, \boldsymbol{R U} \boldsymbol{I}_{\boldsymbol{p}}, \boldsymbol{r T}, \boldsymbol{t}_{\boldsymbol{r}}, \boldsymbol{R U} \boldsymbol{I}_{c}>$
It is ('+') or not ('-') the case that the temporal region at which $R U I_{c}$ considers $n_{i}$
to be an identifying descriptor of the descriptor type $n t_{j}$ for PoR $R U I_{p}$ relates in
manner $r T$ to temporal region $t_{r}$. This tuple type extends the original PtoN-tuple
type from version 1.

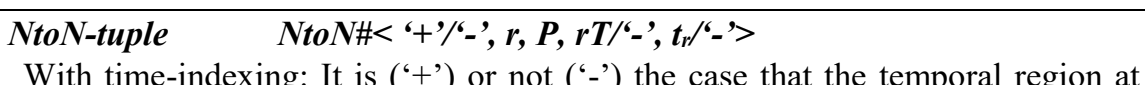
which relation $r$ obtains between the non-repeatable PoRs referred to in the ordered set of RUIs $P$ relates in manner $r T$ to temporal region $t_{r}$.

Without time-indexing: It is ('+') or not ('-') the case that relation $r$ obtains between the non-repeatable PoRs referred to in the ordered set of RUIs $P$.

This tuple type extends the original PtoP-tuple type from version 1.

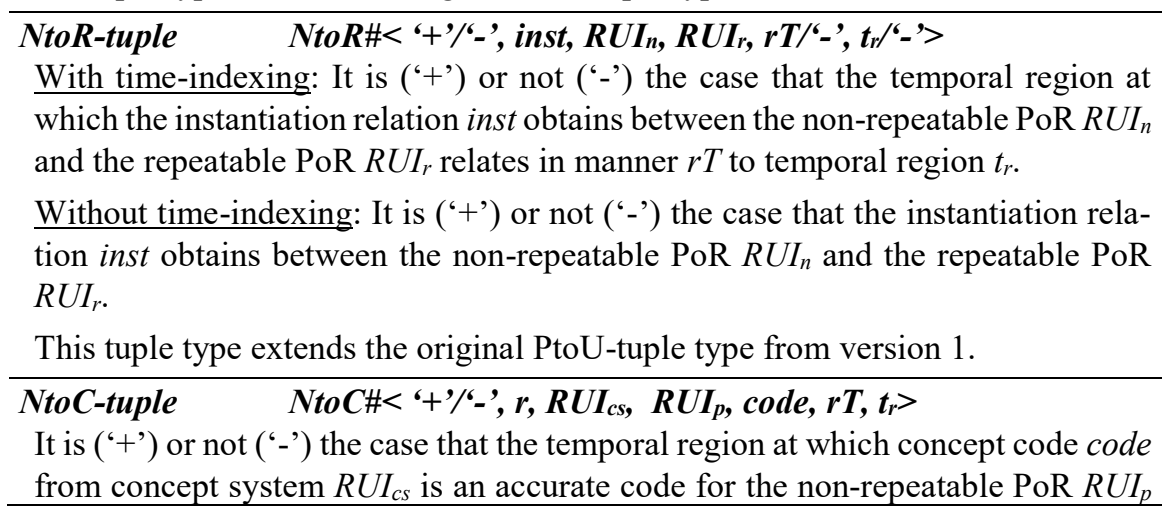


in a way expressed by $r$, relates in manner $r T$ to temporal region $t_{r}$. This tuple type extends the original PtoC-tuple type from version 1.

NtoR(-) -tuple $\quad$ NtoR(-)\#<r, RUI $I_{p}, R U I_{r}, r T /$-' $^{\prime}, t_{r} /$ '-'> $^{-}$

With time-indexing: It is the case that the temporal region at which the relation $r$ does not obtain between the non-repeatable PoR $R U I_{p}$ and any of the non-repeatable PoRs in which repeatable PoR $R U I_{r}$ is repeated relates in manner $r T$ to temporal region $t_{r}$

Without time-indexing: It is the case that the relation $r$ does not obtain between the non-repeatable PoR $R U I_{p}$ and any of the non-repeatable PoRs in which repeatable PoR $R U I_{r}$ is repeated.

This tuple type reflects the original PtoU(-)-tuple type of version 1, authorship however now being declared in a corresponding F-tuple.

It is through A-tuples that unique identifiers are assigned to PoRs that exist or existed, together with the way in which the identifier denotes, i.e. either singularly or probably-not-singularly. RUI reservation is allowed for entities that do not yet exists, but are very likely to come into existence such as the results of blood tests or radiographies that have been ordered. In such cases, a RUI can be 'reserved' rather than 'assigned' [4, 5]. Tuples from any type are automatically assigned a RUI - and can thus themselves be referenced in an RTS to satisfy principle RTP2 - as indicated in table 2 by the tag of the form ' $\mathrm{X \#}$ ' preceding any tuple type. This assignment does not need to be registered by means of an A-tuple as it otherwise would lead to an infinite regress.

D-tuples are used to track the insertion, deletion or correction of tuples. They form together with F-tuples the foundations for mechanisms through which an RTDB can remain faithful through changes in reality and changes in the RTDB itself.

F-tuples are introduced in RT version 2 for two reasons. One is to represent predicates separate from whom authors or supports the predicates what in version 1 was expressed - with the exception of A- and D-tuples which are generated by the RTS itself - as part of the tuples themselves. The second reason is to explicitly represent the confidence supporters of the predicate have in its faithfulness, something not addressed at all in version 1 .

NtoI-tuples serve primarily entity identification needs, i.e. to assess whether some PoR has already been assigned a RUI. These tuples contain annotations which, when appropriately implemented, ensure that querying the RTS for a RUI returns answers which make it possible to determine what the RUI stands for, in part on the basis of the annotations for that RUI, in part on the basis of other RT-tuples in which the RUI is used. An NtoI-tuple can contain the name of the PoR to which ' $R U I_{p}$ ' is assigned, or it can contain a local identifier, a pointer to a photograph, a social security number, and so on. Implementors have the freedom to decide what sorts of identifying information, e.g. demographic information about patients [55], are better suited to be stored in NtoI-tuples than in tuples of other types. Thus the term 'address' can be used as a descriptor type in an NtoI-tuple, the descriptor slot then 
being filled with the actual address of the person in question, or 'address' can be taken from an IAO-compatible ontology and be linked to the person by means of one or more NtoN-tuples in line with the axioms formulated in the ontology.

All remaining tuple types are designed for predicating something about a uniquely identified PoR residing outside the RTDB and which is considered nonrepeatable within the applicable perspective. This is a first major and global change in RT version 2 as compared to version 1 which was exclusively based on the perspective offered by BFO according to which the only non-repeatable PoRs are particulars [9]. As described in section 4.1 the range of non-repeatable PoRs has been extended by taking into account different perspectives.

A second global change introduced in version 2 to tuple types other than A-, D, F- and NtoR(-), is the explicit representation of whether the asserted relationship holds or not, thus allowing for logical negation. A- and D-tuples are excluded because they do not express relations that are outside the RTS. F-tuples are excluded because the degree of confidence or doubt is expressed in the C-slot. The logical negation of a NtoR(-)-tuple - for example one stating that 'the patient has no headache' (formally: 'of all instances of headache, there is none which inheres in this patient within this time frame) - is equivalent to the corresponding affirmative NtoN-tuple, for example stating that 'this instance of diabetes inheres in this patient within this time frame'. That some patient has no headache cannot be expressed using a negated NtoN-tuple because of RT principle RTP1.1 with BFO as overarching ontology: only what exists can uniquely be referenced [10]. Of course, a negated NtoN-tuple in the spirit of this specific example can be made, but then it would convey for instance the triviality of a sort that John Doe's diabetes does not inhere in Jane Smith.

A third change is introduced in the tuple types that in version 1 had a slot for an explicit reference to the ontology from which the expressed relation between PoRs was taken. Since RT has been expanded to work with ontologies other than BFO, some of which offer a realism-based perspective on reality that differs from BFO, it is now required that for each PoR that is referenced by means of an ontology term, this reference includes a reference to the ontology, the version of the ontology and the term.

A fourth change has been introduced by replacing the former PtoC-tuple type by the NtoC-tuple type. In addition to the two global changes discussed above, this tuple type allows now for a broader range of relations than before between a nonrepeatable PoR and a concept or code from a non-realism-based coding or classification system. The former interpretation of tuples of this type was that the terms related to the code, for example what are called 'synonyms' in SNOMED CT, are acceptable to describe something about the PoR to which the code in the tuple is related. Such tuples merely provide a facility equivalent to a simple index of terms in a work of scientific literature [9]. With RT version 2, various relations are allowed on the condition that they are properly defined. One could, for example, define ' $\mathrm{p}$ ofClass $\mathrm{c}$ (at $\mathrm{t}$ )' as a relation which may be used in NtoC-tuples if under a realist interpretation of concept $c$, particular $p$ would be an instance of $c$ (at $t)$. For certain terms from concept-based systems, it is indeed not always clear how they 
need to be interpreted under a realist perspective and what type of entity they precisely denote [2]. In such a case, it would also not be clear what relation should be used to link some phenomenon with the entity it is ascribed to in a NtoN-tuple: inheresIn, participantOf, part of, or what? Yet, when a code $c$ from ICD or SNOMED CT - for instance a code for bone fracture - is used in an EMR, then in the majority of cases it would not be wrong to interpret the annotation as 'patient with some phenomenon for which $c$ is an acceptable code' or 'patient having some phenomenon for which $c$ is an acceptable code' - thus, for example, 'patient with some bone fracture'. Thus for cases like this, one could define for use in NtoNtuples ' $\mathrm{p}$ with $\mathrm{q}$ (at t)' as a rather weak relation expressing that $\mathrm{p}$ experiences (at $\mathrm{t}$ ) some phenomenon $\mathrm{q}$ of which it is stated in another tuple that $\mathrm{q}$ is ofClass $\mathrm{c}$. Caution is still required as this strategy does not work for all codes that might be used in a person's EMR. An example from SNOMED CT would be '256860004 | Fetal brain (substance)' when used in the EMR of a mother as it should not be translated into $\mathrm{RT}$ in a way that implies that the mother has a fetal brain.

\subsection{RT-tuples and faithfulness to reality}

Since RT is anchored in Ontological Realism, RT representations need to be faithful to reality. What this means, specifically in the context of biomedical data, lines up nicely with the data quality constructs defined for the ' $3 \times 3$ DQA evidencebased guideline for electronic health record data reuse' [56]. Table 2 provides a realist interpretation of these constructs, thereby distinguishing between constructs that apply to individual predicates and constructs that assess representations as a whole.

An RT-tuple is faithful to reality whenever it represents veridically, i.e. correctly, the PoR it is about at the level of granularity and from the perspective for which each component of the tuple is designed [57]. An RT-tuple that represents that the tumor in John's stomach was benign in August 2019 is faithful to reality if and only if that was indeed the case, even if that tumor was not 'just a tumor' but a benign polyp, i.e. a specific kind of tumor. The tuple is also faithful throughout reality: even if that polyp turned malignant in April 2020, the veridicality of that tuple is maintained. However, an RT-tuple that states that the tumor in John's stomach was benign since August 2019, would cease to be faithful at the point in time the tumor turned malignant, and this whether or not that change in reality was detected. Where correctness cannot be ascertained, plausibility can be brought in the picture by means of F-tuples.

An RT-tuple is adequately faithful to reality whenever it is faithful to reality at a level of granularity that is equal or more precise than what is required for the purposes for which the tuple is used [53]. A tuple is adequately faithful throughout reality whenever it remains adequately faithful even under changing uses. The coarser the level of granularity at which a tuple represents, the more likely it remains faithful throughout reality. 
A collection of tuples is faithful to reality whenever all of its tuples are faithful, but can be not adequately faithful to reality despite all of its tuples being adequately faithful, and this for several reasons. One reason is that predicates might become outdated, for example when a tumor stated to be benign at some point in time turns malignant, and the purpose of the tuple collection is to be a representation of a patient's medical history. Another reason is that tuples that are individually veridical might be redundant, and this in a variety of ways. An example is predicating at different levels of granularity whereby one tuple is a logical consequence of another because of axioms in the ontology in whose terms they are expressed. Redundancy can also occur because a relationship between two entities is predicated by means of two distinct tuples which contain references to distinct temporal regions of which one is a part of the other. It is therefore necessary that an RTS includes mechanisms to detect unfaithful tuples as well as outdated and redundant ones. This requires maintenance policies and procedures combined with logical reasoning.

Table 2. Quality constructs and related measures for health record data reuse (adapted from [56])

\begin{tabular}{ll}
\hline Construct & Measure \\
\hline $\begin{array}{l}\text { Correctness } \\
\text { Plausibility }\end{array}$ & $\begin{array}{l}\text { Whether a predicate describes its referent faithfully. } \\
\text { Degree to which the body of scientific knowledge provides } \\
\text { arguments for the correctness of a predicate }\end{array}$ \\
$\begin{array}{l}\text { Difference between the time a predicate about some refer- } \\
\text { ent was made and the most recent time at which the referent } \\
\text { exists or existed }\end{array}$ \\
$\begin{array}{l}\text { Degree to which a predicate describes a referent with the } \\
\text { maximal precision obtainable through the observation } \\
\text { method used }\end{array}$ \\
\hline Completeness & $\begin{array}{l}\text { Degree to which predicates about relevant referents are } \\
\text { available }\end{array}$ \\
Foncordance & $\begin{array}{l}\text { Degree to which there is agreement between predicates } \\
\text { Degree to which multiple predicates describe the same fea- } \\
\text { ture of a referent }\end{array}$ \\
Signal-to-noise & $\begin{array}{l}\text { Degree to which irrelevant predicates can be distinguished } \\
\text { from relevant ones }\end{array}$ \\
\hline
\end{tabular}

\subsection{RT tuples and second-hand information}

Assertions in a medical record are about what happened with, or what was or is the case about the patient; they describe, when faithful, the reality on the side of the patient. RT tuples can be used for the same purpose, but have the advantage of of- 
fering a clear and regimented semantics grounded in realism-based ontology. Ideally, any assertion in an EMR would consist exclusively of RT tuples generated at the time the assertion was made. This doesn't mean that clinicians have 'to speak RT'. It would be perfectly possible to modify the user interfaces and back-ends of EMR systems in such a way that the sort of regimentation with which structured data are currently entered can accommodate the generation and storage of RT tuples. It is thereby essential to implement facilities that allow for the explicit and unique identification of all entities on the side of that patient that must exist for the phenomenon denoted by, for example, some ICD or SNOMED CT code, to exist. Figure 1 offers an example using two codes from SNOMED CT, one for a disorder and one for a treatment that a patient with the same sort of disorder might be subjected to. The diagrams which SNOMED CT supplies as representations for the formal definitions for these codes are annotated with placeholders $(\# 1, \# 2, \ldots)$ for RUIs denoting the entities that need to be referenced in RT tuples if the codes would be used in an EMR. SNOMED CT's formal definitions offer - at least in light of the ontology underlying SNOMED CT - a good basis for RT tuples. The RT tuples that would result from it, are able to achieve what neither the codes nor the definitions are able to achieve by themselves: (1) identifying the numerically identical entities that are involved in the context of each code, and not just indicating to what types they belong, and (2) tying the codes together by means of the unique identifiers that appear in both representations (\#1 and \#2).

In the absence of such mechanisms, RT representations of the reality on the side of patients would need to be developed post-hoc by personnel trained in these matters, comparable to how trained clinical coders add, or correct, codes for billing purposes in medical records on the basis of assertions clinicians made, and how Gene Ontology annotations are developed on the basis of the literature describing gene function experiments [58]. The resulting RT tuples, when derived from information a clinician entered in the EMR, would under such scenario be second-hand information. When constructed from billing codes entered by coding clerks, it would even be third-hand information. Because of the provenance principle (RTP2.1), and when relevant for the purposes for which the RTS is designed, such sources need to be represented as well. This may include predicates through which the author of RT tuples expresses his confidence in the reliability of the first-hand information, and predicates about his confidence in the $2^{\text {nd }}$ - and $3^{\text {rd }}$-hand RT tuples resulting from it. While D- and F-tuples are designed to register provenance of and confidence in RT-tuples themselves, they cannot be used to register this information about assertions in the EMR. But because such assertions are parts of reality in their own right, they can be assigned RUIs and even so their authors [59]. This would lead to RT tuples that describe these assertions, the confidence 'post-hoc' authors of the $2^{\text {nd }}$-hand RT tuples have in the faithfulness of the $1^{\text {st }}$-hand assertions, their confidence in a correct interpretation of these assertions, the extent to which they convey the same information as the $1^{\text {st }}$-hand assertions, and so forth. Of course, whether to follow this route depends on the purposes for which the RTS is designed, one such purpose being quality control of EMR-records. 

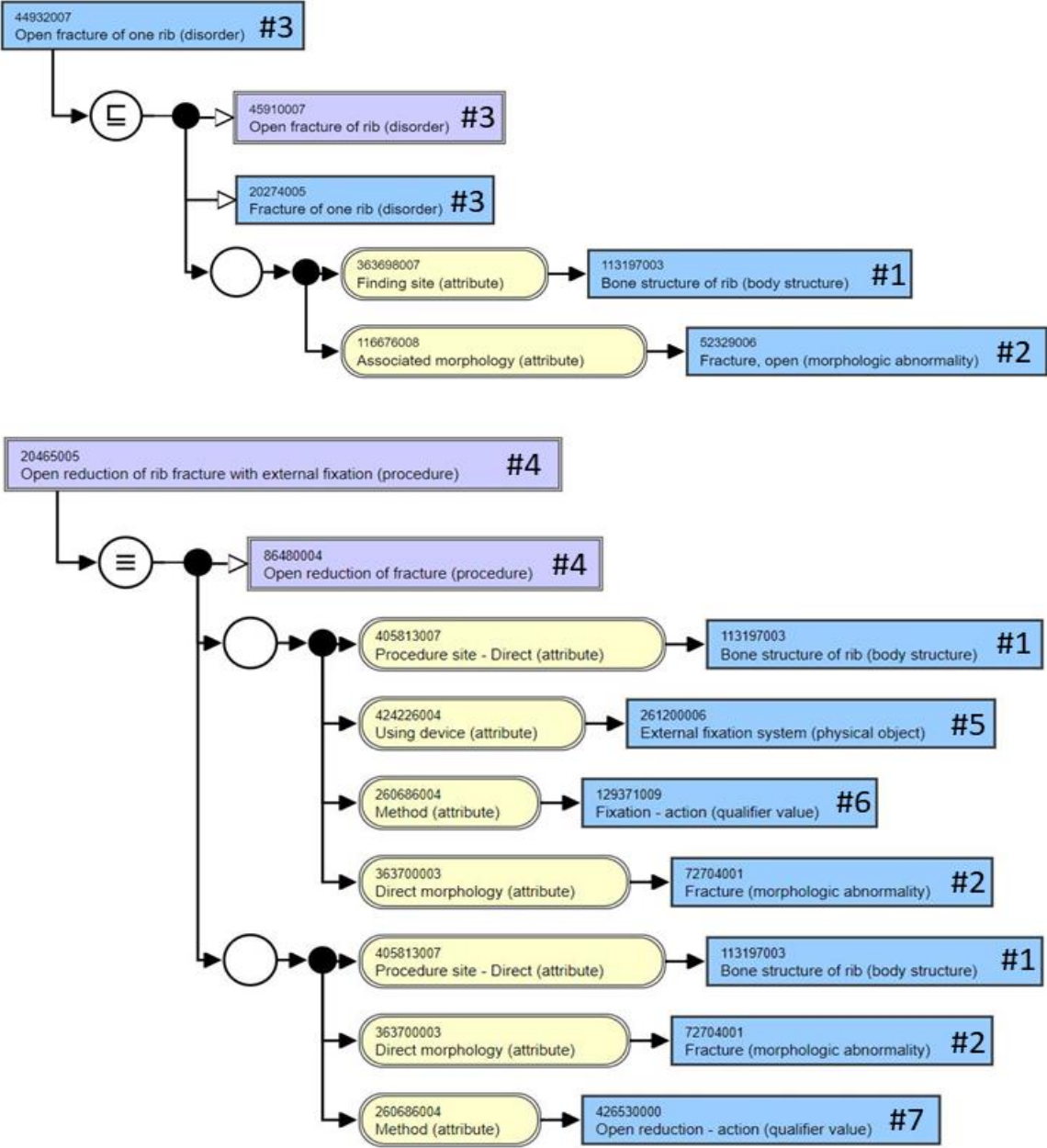

Figure 1. Using SNOMED CT's description logic definitions for concepts as a framework for numerically unique entity identification.

\subsection{Referent Tracking Data Models}

Implementing an RTS requires making a number of choices. One is the selection of appropriate ontologies in whose terms the representations will be made. Some general features of ontologies to be considered were described in section 3. Other features depend on the specific domain to which the RTS will be applied. Further choices to be made concern the mechanisms for creating unique identifiers, assigning them to portions of reality that are uniquely identifiable under the view of the 
chosen ontologies, and retrieving them for portions of reality to which an RT identifier has already been assigned. Also mechanisms to track the referents of the predicates in such a way as to keep the RTDB faithful throughout reality at the desired level of granularity need to be decided upon which will involve decisions as to the type of reasoners to be interfaced with. Finally, choices must be made regarding the sophistication of the mechanisms for representing confidence in F-tuples, types of errors and reasons for change in D-tuples, and whether or not negation will be used in tuples representing reality outside the RTDB.

Decisions on these choices will influence the complexity of the data structure for the RTDB. Implementors are free to choose whatever data structure that satisfies the specifications of the RT paradigm, for instance using for each assertion a structure that mimics the graph structure of the abstract syntax of the appropriate tuple type [60], or storing all information in a relational database [7].

\section{The Problematic Problem List}

To demonstrate the power of RT, let us now turn to a biomedical information management application which is widely considered as being problematic, namely maintaining an accurate problem list in an EMR [12, 61, 62].

Maintaining a problem list in EMRs is in the USA a 'meaningful use' criterion. It was Larry Weed who in the sixties introduced the idea of the problem list for medical record keeping, defining it as 'a complete list of all the patient's problems, including both clearly established diagnoses and all other unexplained findings that are not yet clear manifestations of a specific diagnosis, such as abnormal physical findings or symptoms' [63]. The USA-based meaningful use definition of what counts as a problem is based on a slightly different interpretation of Weed's original conception: it is on the one hand reduced to 'a list of current and active diagnoses ...', thus excluding unexplained findings, while on the other hand stipulating '... as well as past diagnoses relevant to the current care of the patient'. Although this definition sounds simple enough, keeping the problem list complete and accurate is still considered a major problem $[61,64]$.

\subsection{A problem list case study}

A concrete example of this, based on a case study published earlier [12], is provided in table 3 . This table shows for the problem list of the specific patient studied a three-dimensional representation of the data elements persistent in the EMR's back-end database and what portion thereof would be rendered visible in the EMR's user interface at certain points in time. The first dimension - running horizontally covers phenomena on the side of the patient that were assessed as being a medical problem and that were therefor registered on the patient's problem list during the 
time period studied. There are four such problems, each one respectively designated P1 through P4. The second dimension - running vertically - covers the points in time at which the patient's EMR was updated in relation to at least one of these four problems. Time points are represented in the $1^{\text {st }}$ column - marked 'Date' - as number of days since the creation of the problem list at day 1 (November 2011). The table shows that the patient's EMR was updated at ten different points in time over a period of roughly six years and four months in relation to at least one of the four problems. It is of course possible that the EMR was updated more often, but only dates on which updates were made which were explicitly related to one of the problems on the problem list are accounted for in table 3.

The third dimension captures for each problem whether it was involved in an update of the patient's EMR, and if so, in which way. Involvement of a specific problem, when it occurred, in an EMR update at a specific date is depicted in Table 1 at the intersection of the row corresponding to the date and the column corresponding to that problem. When the problem was at that time not yet registered, the intersection is empty. This is for example the case for problems P2, P3 and P4 at day 1 . When there was involvement, the nature of it is represented by means of a vector consisting of eight slots, as for example for problem P1 at day 1 . When the problem was already registered, but is not involved in a specific update, then the slots of the vector are left empty. This is for instance the case for problem P3 at day 979. From here on forward, specific involvements will be referred to by a label formed by combining the abbreviated designation of the problem, e.g. 'P3', with the designation of the day during which the involvement occurred. As an example, the label 'P3-1042' designates the involvement of problem P3 at day 1042. Slots that were changed subsequent to a previous update are depicted in grey background.

Five slots of the involvement vector are used to describe the terminological representation of the problems at different points in time. The slots 'ICD-9', 'ICD-10' and 'Snomed' hold the ICD-9-CM, ICD-10-CM and SNOMED CT codes assigned to the problem, respectively. The 'Term' slot holds the dictionary term assigned to the problem. It is in the dictionary of the EMR system that terms are related to the various codes by means of an associative identifier which is represented in the 'TermDE' slot. The dictionary of the EMR system is regularly updated and this independently from updates to the patient record. On the basis of the history of this patient's problem list, it can be deduced that such an update must have happened between days 979 and 1042 with the introduction of ICD-10-CM and SNOMED CT codes as well as a change in certain terms themselves, i.e. replacing the capitalization of each word in the term by sentence case. This can be seen in problem P2. Another update happened between days 1899 and 2273 with a change in SNOMED CT code for the term assigned to problem P1 and introduction of sentence case for the term assigned to problem P3.

The remaining three slots of the involvement vector represent what sort of involvement precisely occurred at the specified time. The 'Action' slot indicates whether the user performed an intentional action which was directed at the problem. When for a specific involvement, e.g. P1-241, the slot is empty, then there was no 
such action for that problem. Otherwise, the following values qualify the sort of intentional action that happened:

- ' $N$ ', indicating that the problem instance was newly asserted,

- $\quad \mathrm{D}$ ', in case some update in the medical record, e.g. a clinical note or a reference to some investigation or treatment, was performed in relation to the problem,

- ' $\mathrm{R}$ ', when the user declared the problem to be resolved,

- ' $\mathrm{T} \rightarrow \mathrm{Pn}$ ', when the problem was declared to be 'transitioned' into problem $\mathrm{Pn}$,

- ' $\mathrm{T} \leftarrow \mathrm{Pn}$ ', when problem Pn was 'transitioned' into the problem under scrutiny,

- ' $E$ ', when the problem was declared to be entered in error, an involvement that did not occur in this patient's medical history but a possibility offered by the EMR system.

Whenever there is a value in the 'Action' slot, there is also one in the 'Actor' slot, either a unique identifier for the user - here simply $\mathrm{C} 1 \ldots \mathrm{C} 5$, one for each of five distinct persons - who performed the action specified in the 'Action' slot, or a question mark in case no trace for the user could be found in the EMR's back-end database.

The last slot of the involvement vector, 'Status', indicates the status of the problem in relation to the problem list and the actor to whom it applies. Values are expressed using the format ' $\mathrm{X}-\mathrm{C}$ ' where ' $\mathrm{X}$ ' is a symbol for the status proper, and $\mathrm{X}$ for the involved user (or a '?' when the actor is not documented in the EMR system). For involvements tied to an intentional action, the slot reflects the status after the action and this with reference to the actor. The following values are possible:

- 'C' stands for 'created' what occurs either when a problem is added to the problem de novo, e.g. P1-1, or as a transition from another problem, e.g. P4-2317.

- 'D' stands for 'documented' and results from an action of type 'D', e.g. P2-C11.

- 'A' stands for 'archived', something that occurs either as the result of an action of type ' $R$ ', e.g. P3-611, or when the problem is declared to have transitioned into another as in P2-1899.

- 'U' stands for 'updated' and occurs after another problem was transitioned in the one with the ' $U$ ' status, e.g. P1-1899.

- ' $\mathrm{V}$ ' is the status assigned to a problem of which the involvement is not tied to an intentional action involving that problem while it was nevertheless visible to the user while manipulating another problem on the problem list. What is visible in the problem list from a specific update day until a subsequent update day is depicted in bold face in Table 1. This is always the value of the 'Term' slot, and the values of the 'ICD9', 'ICD-10' and 'Snomed' slots when not empty. Since in the EMR system from which our example is drawn a deletion-less transactional 
database approach is implemented, references to archived problems are still retrievable, but are not visualized in the problem list.

Table 3. Evolution of the problem list of a specific diabetic patient in an outpatient EMR.

\begin{tabular}{|c|c|c|c|c|c|c|c|c|c|c|c|c|}
\hline \multirow[b]{4}{*}{ Date } & \multicolumn{3}{|c|}{ Problem P1 } & \multicolumn{3}{|c|}{$\begin{array}{l}\text { Problem P2 } \\
\end{array}$} & \multicolumn{3}{|c|}{ Problem P3 } & \multicolumn{3}{|c|}{ Problem P4 } \\
\hline & Actor & Action & Status & Actor & Action & Status & Actor & Action & Status & Actor & Action & Status \\
\hline & TermDE & Term & & TermDE & Term & & TermDE & Term & & TermDE & Term & \\
\hline & \begin{tabular}{|l|} 
ICD-99 \\
\end{tabular} & ICD-10 & Snomed & ICD-9 & ICD-10 & Snomed & ICD-9 & ICD-10 & Snomed & ICD-9 & ICD-10 & Snomed \\
\hline \multirow{3}{*}{1} & $?$ & $\mathrm{~N}$ & C-? & & & & & & & & & \\
\hline & D1 & \multicolumn{2}{|c|}{ Prediabetes } & & & & & & & & & \\
\hline & 790.29 & & & & & & & & & & & \\
\hline \multirow{3}{*}{241} & & & $\mathrm{~V}-?$ & $?$ & $\mathrm{~N}$ & $\mathrm{C}-?$ & & & & & & \\
\hline & D1 & \multicolumn{2}{|c|}{ Prediabetes } & D2 & \multicolumn{2}{|c|}{ Impaired Fasting Glucose } & & & & & & \\
\hline & 790.29 & & & 790.21 & & & & & & & & \\
\hline \multirow{3}{*}{426} & & & $\mathrm{~V}-\mathrm{Cl}$ & & & $\mathrm{V}-\mathrm{Cl}$ & $\mathrm{C} 1$ & $\mathrm{~N}$ & & & & \\
\hline & D1 & \multirow{2}{*}{\multicolumn{2}{|c|}{ Prediabetes }} & D2 & \multicolumn{2}{|c|}{ Impaired Fasting Glucose } & D3 & \multicolumn{2}{|c|}{\begin{tabular}{|l} 
Diabetes Mellitus \\
\end{tabular}} & & & \\
\hline & \begin{tabular}{|l|}
790.29 \\
\end{tabular} & & & 790.21 & & & 250.00 & & & & & \\
\hline \multirow{3}{*}{611} & & & $\mathrm{~V}-\mathrm{C} 1$ & $\mathrm{C} 1$ & D & D-C1 & $\mathrm{C} 1$ & $\mathrm{R}$ & $\mathrm{A}-\mathrm{Cl}$ & & & \\
\hline & D1 & \multicolumn{2}{|c|}{ Prediabetes } & D2 & \multicolumn{2}{|c|}{ Impaired Fasting Glucose } & D3 & \multicolumn{2}{|c|}{ Diabetes Mellitus } & & & \\
\hline & \begin{tabular}{|l|}
790.29 \\
\end{tabular} & & & 790.21 & & & 250.00 & & & & & \\
\hline \multirow{3}{*}{979} & $\mathrm{C} 2$ & $\mathrm{D}$ & $\mathrm{D}-\mathrm{C} 2$ & & & $\mathrm{~V}-\mathrm{C} 2$ & & & & & & \\
\hline & D1 & \multicolumn{2}{|c|}{ Prediabetes } & D2 & \multicolumn{2}{|c|}{ Impaired Fasting Glucose } & & & & & & \\
\hline & \begin{tabular}{|l|}
790.29 \\
\end{tabular} & & & 790.21 & & & & & & & & \\
\hline \multirow{3}{*}{1042} & & & $\mathrm{~V}-\mathrm{C} 1$ & $\mathrm{C} 1$ & $\mathrm{D}$ & D-C1 & & & $\mathrm{A}-\mathrm{C} 1$ & & & \\
\hline & $\mathrm{D} 4$ & \multicolumn{2}{|c|}{\begin{tabular}{|l} 
Prediabetes \\
\end{tabular}} & D5 & \multicolumn{2}{|c|}{ Impaired fasting glucose } & D6 & Diabetes & Mellitus & & & \\
\hline & \begin{tabular}{|l|l|}
790.29 \\
\end{tabular} & $\begin{array}{l}\text { R73.09 } \\
\end{array}$ & 9414007 & 790.21 & R73.01 & 390951007 & 250.00 & \begin{tabular}{|l|l|} 
E11.9 \\
\end{tabular} & 73211009 & & & \\
\hline & $\mathrm{C} 3$ & $\mathrm{D}$ & D-C3 & & & $\mathrm{V}-\mathrm{C} 3$ & & & & & & \\
\hline 1720 & D4 & Prediabet & & D5 & Impaired fa & ting glucose & & & & & & \\
\hline & 790.29 & R73.09 & 9414007 & 790.21 & R73.01 & 390951007 & & & & & & \\
\hline & & & $\mathrm{V}-\mathrm{C} 4$ & $\mathrm{C} 4$ & D & D-C4 & & & & & & \\
\hline 1801 & D4 & Prediabet & & D5 & Impaired fa & ting glucose & & & & & & \\
\hline & 790.29 & R73.09 & 9414007 & 790.21 & R73.01 & 390951007 & & & & & & \\
\hline & $\mathrm{C} 4$ & $T \leqslant P 2$ & U-C4 & $\mathrm{C} 4$ & $\mathrm{~T} \rightarrow \mathrm{P} 1$ & A-C4 & & & & & & \\
\hline 1899 & D4 & Prediabet & & D5 & Impaired fas & ng glucose & & & & & & \\
\hline & 790.29 & \begin{tabular}{|l|} 
R73.09 \\
\end{tabular} & \begin{tabular}{|l|l|}
9414007 \\
\end{tabular} & 790.21 & R73.01 & 390951007 & & & & & & \\
\hline & $\mathrm{C} 4$ & $\mathrm{~T} \rightarrow \mathrm{P} 3$ & $\mathrm{~A}-\mathrm{C} 4$ & & & & $\mathrm{C} 4$ & $T<P 1$ & U-C4 & & & \\
\hline 2273 & D8 & Prediabete & & & & & D6 & Diabete & s mellitus & & & \\
\hline & 790.29 & R73.09 & 714628002 & & & & 250.00 & \begin{tabular}{|l|} 
E11.9 \\
\end{tabular} & 73211009 & & & \\
\hline & & & & & & & C5 & $\mathrm{T} \rightarrow \mathrm{P} 4$ & A-C5 & $\mathrm{C} 5$ & $T \leftarrow P 3$ & C-C5 \\
\hline 2317 & & & & & & & D6 & Diabetes & s mellitus & D7 & \begin{tabular}{|l|} 
Type II DM \\
\end{tabular} & vell controlled \\
\hline & & & & & & & 250.00 & \begin{tabular}{|l|l|} 
E11.9 \\
\end{tabular} & 73211009 & 250.00 & E11.9 & 44054006 \\
\hline
\end{tabular}

\subsection{Does this problem list provide answers or raise questions?}

The reality, of course, is both. At first sight and from a mere clinical point of view, there seems to be nothing extraordinary about the problem list of this patient as it shows a transition from prediabetes into diabetes, an evolution which is estimated to occur from $5 \%$ to $10 \%$ in the roughly 400 million prediabetes patients worldwide [65]. But from a biomedical data science perspective towards the secondary use of these data for clinical research or decision support, matters are much less clear. This is not only the case for the problem list used here as example, rather it seems to be a structural problem of EMRs in general and data repositories collated through common data models such as OMOP, i2b2 or PCORNet which distort information even further [12-16, 66-69].

The issues with this particular problem list - and even with the very idea of 'problem list' in general as will be demonstrated - become quite clear when one attempts to scrutinize the individual data elements on the basis of the data quality constructs listed in table 2 thereby resorting to the closely related meanings of the 
various classification codes used. How the codes used in the problem list relate to each other as well as to other codes in the respective coding and classification systems is shown in table 4.

Table 4. Comparison of ICD-9-CM, ICD-10-CM and SNOMED CT diabetes related terms for codes used in Table 3 .

\begin{tabular}{|c|c|c|}
\hline $\begin{array}{c}\text { ICD-9 } \\
\text { ICD-10 }\end{array}$ & Index & $\begin{array}{l}\text { ICD-term } \\
\text { SNOMED code }\end{array}$ \\
\hline $\begin{array}{l}790.21 \\
\text { R73.01 }\end{array}$ & $\begin{array}{l}\text { A1 } \\
\text { A1a }\end{array}$ & $\begin{array}{cc}\text { Impaired/elevated fasting glucose } \\
390951007 & \text { Impaired fasting glycemia (disorder) }\end{array}$ \\
\hline $\begin{array}{r}790.22 \\
\text { R73.02 }\end{array}$ & $\begin{array}{l}\text { B1 } \\
\text { B1a } \\
\text { B1b } \\
\text { B1c }\end{array}$ & $\begin{array}{cl}\text { Impaired/elevated glucose tolerance test } \\
274858002 & \text { Abnormal glucose tolerance test (finding) } \\
166927002 & \text { Impaired glucose tolerance test (finding) } \\
9414007 & \text { Impaired glucose tolerance (disorder) }\end{array}$ \\
\hline $\begin{array}{c}790.29 \\
\mathrm{R} 73.03 \\
(\mathrm{R} 73.09)^{1}\end{array}$ & $\begin{array}{l}\text { C1 } \\
\text { C1a } \\
\text { C2 } \\
\text { C2a } \\
\text { C3 } \\
\text { C4 }\end{array}$ & 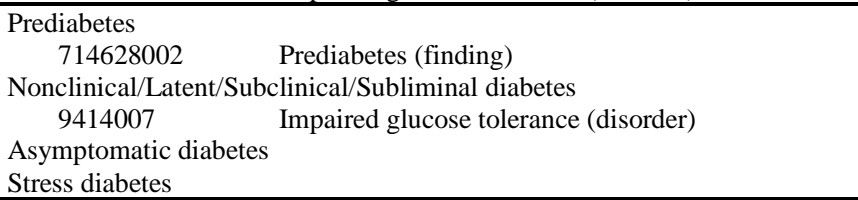 \\
\hline $\begin{array}{l}790.29 \\
R 73.09\end{array}$ & $\begin{array}{l}\text { D1 } \\
\text { D1a } \\
\text { D2 } \\
\text { D2a } \\
\text { D2b } \\
\text { D3 } \\
\text { D3a } \\
\text { D4 } \\
\text { D4a } \\
\text { D5 } \\
\text { D5a } \\
\text { D6 }\end{array}$ & 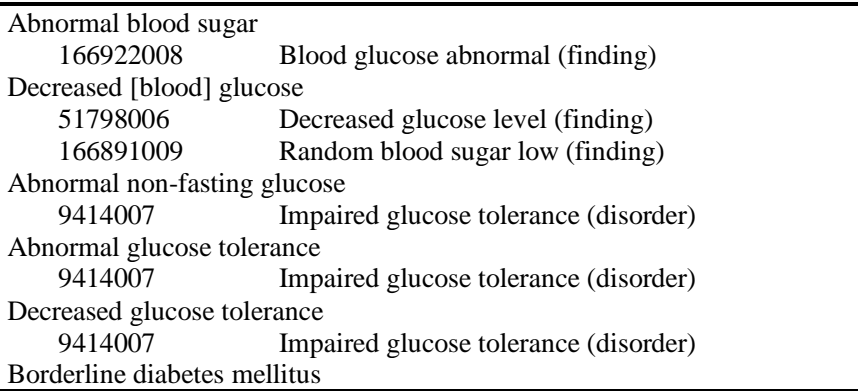 \\
\hline $\begin{array}{l}790.29 \\
\mathrm{R} 73.9\end{array}$ & $\begin{array}{l}\text { E1 } \\
\text { E1a } \\
\text { E2 } \\
\text { E2a } \\
\text { E3 }\end{array}$ & 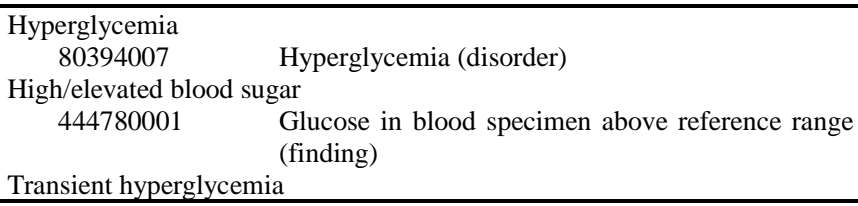 \\
\hline $\begin{array}{c}250.00 \\
-\end{array}$ & $\begin{array}{l}\text { F1 } \\
\text { F1a } \\
\text { F1b } \\
\text { F1c } \\
\text { F1d } \\
\text { F1e }\end{array}$ & $\begin{array}{cc}\text { Diabetes mellitus without mention of complication, type II or unspecified } \\
\text { type, not stated as uncontrolled } \\
73211009 & \text { Diabetes mellitus (disorder) } \\
44054006 & \text { Diabetes mellitus type 2 (disorder) } \\
199230006 & \text { Pre-existing type 2 diabetes mellitus (disorder) } \\
472969004 & \text { History of diabetes mellitus type 2 (situation) } \\
444110003 & \text { Type II diabetes mellitus well controlled (finding) }\end{array}$ \\
\hline $\begin{array}{c}250.00 \\
\text { E11.9 }\end{array}$ & $\begin{array}{l}\text { G1 } \\
\text { G1a }\end{array}$ & $\begin{array}{ll}\text { Type } 2 \text { diabetes mellitus without complications } \\
\begin{array}{ll}\text { Type II diabetes mellitus without complication (dis- } \\
\text { order) }\end{array}\end{array}$ \\
\hline
\end{tabular}

${ }^{1}$ ICD-10-CM code 'R73.03' was introduced in 2020. Prior to that, the terms associated with it were to be coded as 'R73.09'.

So, what questions does this problem list raise? A first one would be whether at any point in time that more than one problem is active, the list needs to be assessed 
as exhibiting the highest quality with respect to granularity, concordance, fragmentation and signal-to-noise ratio. And if that is not the case, whether it is an issue of correctness or inadequate granularity, the latter either in terms of a more specific or generic type ('type-granularity', e.g. 'diabetes mellitus' versus 'diabetes mellitus type I'), or in terms of 'part-whole granularity', e.g. 'cheek' versus 'face').

From days 241 to 1899 , for example, both prediabetes (P1) and impaired fasting glucose (P2) are noted as distinct active problems while impaired fasting glucose (IFG) is one of the diagnostic features of prediabetes [70]. There is thus undeniably concordance between the two predicates, but also increased fragmentation and reduced signal-to-noise ratio. The fragmentation - two registered problems are in reality only one problem - has the untoward consequence that updates on the patient's condition were fragmented as well: where users C2 and C3 linked their documentation to $\mathrm{P} 1$, users $\mathrm{C} 1$ and $\mathrm{C} 4$ did so to $\mathrm{P} 2$. When at day $1899, \mathrm{P} 2$ is predicated by $\mathrm{C} 4$ to be transitioned into $\mathrm{P} 1$, seemingly to eliminate the fragmentation, that very act introduces two new mistakes with respect to currency and correctness: indeed, it was not from day 1899 on that IFG 'became' prediabetes - that is what 'transitioning' means in this particular EMR system - but it was already a feature of prediabetes from the very beginning, though perhaps not discovered because not tested for.

Matters become worse at day 426 when diabetes mellitus is also brought into the mix as a third concurrent active problem, thus ending up with a patient about whom it seems to be discordantly predicated that he has prediabetes and diabetes mellitus at the same time. One might object to this assumption by arguing that the time at which the predicates are made is what really counts: it was a problem that the patient had prediabetes at day 1 , something which was reconfirmed at day 421 , and that at day 426 the problem is diabetes. But what is then the meaning of 'active' in the term 'active problem list'? The meaningful use interpretation allows any diagnosis made in the past to be included in the active problem list when it is still relevant for the current care of the patient, but then, is it really relevant for the care of a diabetes patient that he had prediabetes in the past?

Another question arises when the patient's diabetes is predicated by user $\mathrm{C} 1$ to be 'resolved' on day 611. Was the patient's diabetes really cured - the likelihood of this having happened is extremely low [71] and indeed, at day 2273 diabetes is added to the patient's problem list again - or does the predicate merely reflect that at that point in time the patient's glucose levels tested normal? There are several issues here. One issue is the homonymous use, for instance by the American Diabetes Association [72, Figure 1, p.S82], of the term 'diabetes mellitus' for the disease itself as well as for a later stage of the disease during which a fasting plasma glucose of $\geq 7.0 \mathrm{mmol} / \mathrm{l}$ or an $\mathrm{HbAlc}$ of $\geq 48 \mathrm{mmol} / \mathrm{mol}$ is found [73]. Another issue is the confusion between symptom and disease on the one hand, and disease and diagnosis on the other hand, both being painfully apparent in the ICD family of classifications. Although 'ICD' stands for 'International Classification of Diseases', several codes do not indicate diseases at all, but rather symptoms or signs (table 4). Furthermore, most codes from ICD may only be used when certain diagnostic criteria are met, but clearly, when a patient has a disease such as type 2 diabetes as 
demonstrated in the past by a fasting plasma glucose test above the stated criteria, then the disease does not disappear when at a later time a blood glucose test does not meet the diagnostic criteria. Taking all this in consideration, then a better approach for day 611 would have been to create a new problem P4, mark it as wellcontrolled type 2 diabetes mellitus (ICD-9: 250.00) and transition P1, P2 and P3 into it, thus ending up with a problem list already at day 611 of the form it originally obtained only more than three years later!

Issues with respect to granularity become clear as soon as SNOMED CT is additionally used to code problems starting at day 1042. The ICD-codes 790.22/R73.02 for 'Impaired/elevated glucose tolerance test' (Index B1 in table 4) run together three distinct codes in SNOMED CT which distinguishes, first, 'impaired' from 'abnormal' - though, granted, with no explanation for this difference although one could argue that having an 'impaired' glucose tolerance test is not 'abnormal' for a diabetic patient - and second, the result of the test, a 'finding', from what SNOMED CT considers to be the disorder responsible for the impaired test result. But at the same time, SNOMED CT accepts 'latent diabetes' as a synonym for 'impaired' glucose tolerance (disorder)' while for ICD 'latent diabetes' is synonym for 'prediabetes' (Index $\mathrm{C}$ in table 4). The consequence is that picking this term from SNOMED CT may lead to two distinct codes in ICD: 790.22/R73.02 or 790.29/R73.09.

One last question addressed here concerns the extent to which the provenance of the predicates is accurate, specifically for the assignment of the codes. Table 3 shows that ICD-10-CM and SNOMED codes were added to the problems at day 1042. Yet, it is only with respect to problem P2 that the patient's record was updated by user $\mathrm{C} 1$. P3 was already archived and not touched while P1 was visible to $\mathrm{C} 1$ but also not touched. Nevertheless, new codes were assigned, not by user $\mathrm{C} 1$ as is erroneously asserted in the back-end database, but through a procedure running in the background after an update to the dictionaries used by the EMR system. That is also the case for the SNOMED-CT code change of problem P1 at day 2273.

\subsection{A path to improvement}

Clearly, the problem list used as example, the information model according to which the relevant part of the EMR's back-end database is built, and the way in which the data elements for the problem list are persisted to the database do not satisfy all the predication principles described in section 1 to which Referent Tracking adheres to. Unique identification (RTP1.1) is maintained for the patient, but only partly for the authors of the predications and not at all for the individual entities the predications are about. Hence the ambiguity in whether certain predicates are about the disease (type 2 diabetes) the patient is suffering from, a phase of that disease, or phenomena such as a high blood glucose level after fasting which are caused by the disease. The EMR system does keep track most of the time by whom the predications were made and all the time when they were asserted. Confirmation 
of the existence of a problem at a later time is implicitly confirmed for those problems to which documentation is added (RTP2.1). Contradictions, though existing in this example, are not explicitly tracked. The EMR system from which the problem list was taken allows for making corrections but no examples of this were found here.

The EMR system does not use an overarching ontology to represent the reality on the side of the patient (RTP3). Structured representations are coded in ICD-9/10 both of which lack an ontology and SNOMED CT which does have one, be it crippled in many ways $[25,26]$. Yet, it is precisely through the appropriate use of ontology that almost all issues discussed above could have been prevented if the EMR system were appropriately designed. Merely picking terms from a realism-based ontology rather than from ICD or SNOMED CT wouldn't do the trick. What would be required is that the EMR would follow sound ontological principles so that clinicians are able to register data in a way that not only satisfies the $3 \times 3$ DQA evidence-based guideline for electronic health record data reuse [56] but also other best practice guidelines, and this without the need for the EMR user to have deep knowledge of such guidelines. Ideally, that would be achieved through a complete redesign of the information model in line with these ontological principles, and as a consequence also of the back-end database, business rules and user interfaces. A less drastic but equally valid alternative would be to adjust the user interfaces of the EMR system so that the data captured - whether for data registration or for querying the data already registered - are processed further in two data streams: one to/from the existing back-end database and one to/from an RTS.

\section{$7 \quad$ Problem List Management through Referent Tracking}

The principles of RT and the abstract syntax and semantics of RT representations being explained, and the issues in the problem list used as example being pointed out, the question is now to what extent it would be possible to translate the problem list into an RT representation which offers an adequately faithful view on that part of the patient's medical history which is described in the problem list. This question will be answered using a 'post-hoc' scenario under which a newly installed RTS would be interfaced with the EMR system at a time just after the last change to this problem list. This allows to address also a second question: could some of the issues with this problem list have been prevented if the EMR system under an 'integrated' scenario had adopted the RT principles from the very beginning?

The first step to achieve this objective is to find - and (if needed) to adapt or develop - ontologies, or foundations for such ontologies, that have sufficient coverage for the intended representation. The second step is to set up a data structure that can accommodate the semantics of the RT tuple types. The third step is to identify the numerically singular entities to which predicates need to be applied, denote these entities by means of RUIs, and use the RUIs in predicates that describe the 
underlying reality of the problem list in terms of the chosen ontologies and in an RT-compatible way.

The RT-compatible representation provided below will be incomplete and only scratch the surface of what can and ought to be done. Yet, the representation will be detailed enough to demonstrate the advantages and opportunities of the RT approach while highlighting the difficulties one can be confronted with when attempting to identify what, and about what, clinicians exactly are predicating when entering data in an EMR.

\subsection{Ontologies for the problem list}

Although other options exist, the representation proposed here is entirely based on BFO-derived ontologies and ontology-related work that fits that framework.

There is thus far no ontology that is specifically designed for problem list annotation. Building one for that purpose would currently be premature for the simple reason that the notion of 'problem' as described by Weed and in works derived there from is too sketchy to be a workable basis for a formally well-structured ontology. While in the course of nearly a century, philosophers have been giving a variety of accounts as to the general notion of 'problem', each one focused however on one specific aspect, it is only very recently that an attempt has been made to come to an overarching characterization as 'a state of affairs or situation in which something valued is harmed or is obstructed from reaching an end both valued and assigned to it' $[74, \mathrm{p} 8]$, though without offering a formal ontology.

What in the medical domain comes close to a 'medical problem ontology' is the CORE subset of SNOMED CT [75]. This subset is a collection of SNOMED CT concepts that are drawn from the hierarchies 'Clinical finding' (which includes 'Disorders'), 'Procedure', 'Situation with explicit context', and 'Events' and that are sensible candidates for being used as elements of the problem list. It is in some respect a better tool than SNOMED CT itself for this purpose: SNOMED CT does have the concept '55607006 | Problem (finding)' under which there are in the International Version 2020073124 direct subclasses which further expand in about 120 more specific concepts. Yet there are in that 457 concepts which have the word 'problem' in their fully specified name, many of which, for example '290119008 | Nipple problem (finding)', are not subsumed by '55607006 | Problem (finding)'. A disadvantage of SNOMED CT, and thus of any subset thereof, is that it is conceptbased, thus focusing on 'meanings' rather than on what terms are about, and that the underlying ontology does not do justice to the crucial distinctions discussed in section 3.2. It is - at least for a metaphysical and scientific realist - obvious that the discrepancy in SNOMED CT between what is terminologically labelled as a problem and what is - or not - formally classified as a problem, is caused by a confusion between phenomena on the side of the patient and representations thereof such as conclusions based on the assessment of these phenomena. This type of flaw has become pervasive in medical informatics mainly through the now obsolete HL7 
RIM [76, 77] which exhibited a line of confused thinking which is still practiced in its successor FHIR which claims on the one hand that a condition is ' a clinical condition, problem, diagnosis, or other event, situation, issue, or clinical concept that has risen to a level of concern' and on the other hand that 'conditions are frequently a result of a clinician's assessment' [78]. Fortunately, these issues can be accommodated through RT-tuples in combination with appropriate BFO-based ontologies.

An adequate ontology is the Ontology for General Medical Science (OGMS) [23] in combination with the Ontology for Biomedical Investigations (OBI) [24] since both contain well-defined universals which are instantiated by PoRs on the side of the patient of the sort which are documented on the problem list. Not suited is the Disease Ontology (HDO) which despite being accepted as an OBO Foundry ontology [79] follows BFO only very superficially and OGMS not at all: its terms are not intended to denote universals but rather 'disease concepts' [80, p584]. The Monarch Disease Ontology (MONDO) is also unacceptable as it does not distinguish between disorders and diseases and does not use any upper ontology [81]. Of course, terms from both HDO and MONDO can be used in NtoC-tuples in the same way as terms from SNOMED CT or ICD.

Universals well-defined in OGMS and relevant for the problem list used as example here are:

- Disorder $=$ def. - A causally relatively isolated combination of physical components that is (a) clinically abnormal and (b) maximal, in the sense that it is not a part of some larger such combination.

- Pathological Process $=$ def. - A bodily process that is a manifestation of a disorder.

- Disease $=$ def. - A disposition (i) to undergo pathological processes that (ii) exists in an organism because of one or more disorders in that organism.

- Disease Course $=$ def. - The totality of all processes through which a given disease instance is realized.

- Laboratory Finding = def. - A representation of a quality of a specimen that is the output of a laboratory test and that can support an inference to an assertion about some quality of the patient.

- Manifestation of a Disease $=$ def. - A bodily process, bodily quality or bodily component of a patient that is (a) a deviation from clinical normality that exists in virtue of the realization of a disease and (b) is observable.

Relevant universals from OBI are 'organism' and its descendant 'Homo sapiens' (an NCBI taxon term imported into OBI). Although the NCBI taxonomy uses the term 'homo sapiens' to denote the unique species to which human beings belong homo sapiens is in this sense thus an instance of the universal 'species' - one can assume that OBI does not intend to denote with that term the species 'homo sapiens' - after all, a species is not a kind of organism - but the defined class formed by all instances of the universal 'organism' which belong to the species 'homo sapiens' [60].

Also required are certain elements that form the foundations for the Information Artifact Ontology [21]. Thus far, the word 'representation' has been used rather 
informally in this chapter. Formally, a representation is defined as a quality that is about - or is intended to be about $-\mathrm{a}$ PoR [21]. A consequence of this definition is that, formally, it would be incorrect to say, for example, that a discharge letter is about some patient; the correct way would be to say that there exist some quality $q$ that inheres in the discharge letter and that it is $q$ which is about that patient. Furthermore, each representation $q$ concretizes some instance of information content entity $p$, and $p$ may be concretized in many other representations than $q$. The relation synonymousWith can then be defined in such a way that it can be used to state that some RT tuple denotes the same PoR as intended by the use of some ICD or SNOMED CT code in the EMR.

\subsection{Choice of data structures}

RT tuples will be represented below in table records that follow closely the format of the corresponding tuple types as described in Table 1, one table for each tuple type. Each A-, D- and F-record follows the format of the corresponding tuples exactly, the first slot thereby containing the RUI for the information content entity which is concretized in the record. Negative assertions will not be used, thus the '+'/'-' slot will be omitted and all records to be interpreted as statements of the form 'it is the case that ...'. All relations will be ternary or binary depending on whether time-indexing is required. Therefore, NtoN-records will not contain in the P-slot a list of RUIs denoting the relata, but a RUI for a single PoR. The order of slots in the tuple types used to denote relations and their relata will be changed to produce the better readable format ' $p$ relatedTo $q$ (at $t)$ ' in the corresponding records.

A number of notational conventions will be used to make it easier to identify what the RUIs in each of the records stand for. RT table records will continue to be uniquely identified following the format ' $\mathrm{X} \# \mathrm{n}$ ' where ' $\mathrm{X}$ ' is the name of the tuple type, and ' $n$ ' an integer. Particulars external to the RTS will be uniquely identified through the format ' $\#$ n' with the exception of temporal regions for which 'tn' will be used, 't' being a literal and ' $n$ ' an integer. Exceptions are temporal regions that correspond to time intervals of which the temporal boundaries are dates explicitly referenced in the problem list. Because the population of the RTDB will be carried out under the assumption that the RTS is newly installed, the counters for all tables, as well as for the PoRs outside the RTS, will start at ' 1 '.

Relations will be written in full and primarily be taken from the BFO standard ISO/IEC 21838-2 or the sources the standard originates from as well as defined in the foundational papers for the selected BFO-compatible ontologies [20-24]. Both relations and types will be preceded by the ontology drawn from, as, for example, in 'BFO:Object'. Relations that are not prefixed are to be interpreted as being defined in the RTS. This will also be the case for the temporal relations defined in ISO-standard 12381:2019. 
Terms taken from concept-based ontologies and terminologies, as well as from classification systems, will be of the form ' $\mathrm{X}: \mathrm{c}$ ' where ' $\mathrm{X}$ ' is the name or abbreviation for the resource, and 'c' the code for the concept or class the term is associated with.

Although each record is identified by a RUI, an additional sequentially numbered denotation of the form ' $r n$ ' - where ' $n$ ' is an integer - will appear on the right of each record as an additional index for easy reference. Finally, whenever a RUI is written between quotes, the RUI is mentioned, while without quotes it is used as proxy for the real-world entity to which it is assigned [82].

\subsection{Core actors}

The first question to be answered is 'who are the core actors that need to be represented and therefore must be assigned a RUI?'. Under a post-hoc scenario involving the installation of an empty RTS, the first RUI must be assigned to the person setting up the system, here Jane Smith, who is also assumed to carry out the analysis and to populate the RTDB with the problem list related tuples. Then there are the five clinicians (referenced in table 3 as $\mathrm{C} 1$ to $\mathrm{C} 5$ ), and the patient (John Doe), all assumed here to be distinct persons. A problem under the post-hoc scenario that could have been avoided when the EMR system would have been serviced by an RTS from the very start is that the EMR does not identify the author(s) of problemlist entries P1-1 and P2-241. The appropriate approach is here to generate a RUI for each author but thereby annotating both RUIs in their A-record as not being guaranteed to be singularly unique. Up to this point, the A-table would look like this, assuming the assignments to have been made at time 2020-04-05T14:30Z:

\begin{tabular}{|c|c|c|c|c|c|}
\hline A\# & $\boldsymbol{R} U \boldsymbol{I}_{a}$ & $\boldsymbol{R U} \boldsymbol{I}_{p}$ & 'A ${ }^{\prime} /{ }^{\prime} R$ ' & ${ }^{\top}+S U^{\prime} /{ }^{-}-S U^{\prime}$ & $t$ \\
\hline A-1 & $\# 1$ & $\# 1$ & A & $+\mathrm{SU}$ & 2020-04-05T14:30Z \\
\hline A-2 & $\# 1$ & \#2 & A & $+\mathrm{SU}$ & 2020-04-05T14:30Z \\
\hline A-3 & $\# 1$ & $\# 3$ & A & $+\mathrm{SU}$ & 2020-04-05T14:30Z \\
\hline A-4 & $\# 1$ & \#4 & A & $+\mathrm{SU}$ & 2020-04-05T14:30Z \\
\hline A-5 & $\# 1$ & $\# 5$ & A & $+\mathrm{SU}$ & 2020-04-05T14:30Z \\
\hline A-6 & $\# 1$ & \#6 & A & $+\mathrm{SU}$ & 2020-04-05T14:30Z \\
\hline A-7 & $\# 1$ & \#7 & A & $+\mathrm{SU}$ & 2020-04-05T14:30Z \\
\hline A-8 & $\# 1$ & $\# 8$ & A & $-\mathrm{SU}$ & 2020-04-05T14:30Z \\
\hline A-9 & $\# 1$ & \#9 & A & $-\mathrm{SU}$ & 2020-04-05T14:30Z \\
\hline
\end{tabular}

The NtoI-table could at this point on the basis of the information available look something like this:

$\begin{array}{llllrrrr}\text { NtoI\# } & \boldsymbol{R} \boldsymbol{U} \boldsymbol{I}_{\boldsymbol{p}} & \boldsymbol{n t}_{\boldsymbol{j}} & \boldsymbol{n}_{\boldsymbol{i}} & \boldsymbol{r} \boldsymbol{T} & \boldsymbol{t}_{\boldsymbol{r}}, & \boldsymbol{R} \boldsymbol{U} \boldsymbol{I}_{\boldsymbol{c}} & \\ \text { NtoI-1 } & \# 1 & \text { Name } & \text { Jane Smith } & \text { at } & \text { t1 } & \# 1 & (\mathrm{r} 10) \\ \text { NtoI-2 } & \# 2 & \text { Staff ID } & \text { C1 } & \text { at } & \text { t2 } & \# 1 & \text { (r11) }\end{array}$




$\begin{array}{llllllll}\text { NtoI-3 } & \# 3 & \text { Staff ID } & \text { C2 } & \text { at } & \text { t3 } & \# 1 & \text { (r12) } \\ \text { NtoI-4 } & \# 4 & \text { Staff ID } & \text { C3 } & \text { at } & \text { t4 } & \# 1 & \text { (r13) } \\ \text { NtoI-5 } & \# 5 & \text { Staff ID } & \text { C4 } & \text { at } & \text { t5 } & \# 1 & \text { (r14) } \\ \text { NtoI-6 } & \# 6 & \text { Staff ID } & \text { C5 } & \text { at } & \text { t6 } & \# 1 & \text { (r15) } \\ \text { NtoI-7 } & \# 7 & \text { Name } & \text { John Doe } & \text { at } & \text { t7 } & \# 1 & \text { (r16) } \\ \text { NtoI-8 } & \# 8 & \text { Ref } & \text { Actor P1-1 } & \text { at } & \text { t8 } & \# 1 & \text { (r17) } \\ \text { NtoI-9 } & \# 9 & \text { Ref } & \text { Actor P2-241 } & \text { at } & \text { t9 } & \# 1 & \text { (r18) }\end{array}$

RTS implementors have the freedom to use the $\boldsymbol{n} \boldsymbol{t}_{j}$ attributes in this table as they see fit for the purposes of the application. It would be possible, for instance, to use for each person more than one NtoI-record and separate names in their constituent parts such as 'first name', 'last name', etc., or, as shown here for the clinicians, use other references such as identifiers assigned by the facility. This kind of information could also be expressed by means of NtoN-records but there is no point in doing so without a use case for logical reasoning with names or medical record numbers, although, of course, names and medical record numbers are real-world entities themselves.

The 'at' in the $\boldsymbol{r} \boldsymbol{T}$ position is a 'maximal at' which in RT is defined in line with ISO-standard 12381:2019 as specifying the maximal temporal region during which a relationship holds. In the context of NtoI-records, this is the maximal temporal region during which the identifying descriptor in the $\boldsymbol{n}_{\boldsymbol{i}}$ slot was, is and will be appropriate for the entity denoted in the $\boldsymbol{R} \boldsymbol{U I}_{\boldsymbol{p}}$ slot. This is different from the use of 'at' in $\mathrm{BFO}$ where it means rather at least during the specified temporal region: the relationship between the entities must hold during the entire referenced temporal region, but may in addition hold before or after the referenced temporal region.

For all A- and NtoI-records created thus far, corresponding D-records had to be inserted, as for example displayed here for $\mathrm{r} 1, \mathrm{r} 10, \mathrm{r} 2$, and $\mathrm{r} 11$ respectively:

$\begin{array}{llllrrr}\text { D\# } & \boldsymbol{R} \boldsymbol{U} \boldsymbol{I}_{\boldsymbol{d}} & \boldsymbol{R} \boldsymbol{U} \boldsymbol{I}_{\boldsymbol{T}} & \boldsymbol{T} & \boldsymbol{I}{ }^{\prime} \boldsymbol{E} & \boldsymbol{R} & \boldsymbol{S} \\ \text { D-1 } & \# 1 & \text { A-1 } & 2020-04-05 \mathrm{~T} 14: 30 \mathrm{Z} & \mathrm{I} & \mathrm{A}+2 / \mathrm{P}+1 & - \\ \text { D-2 } & \# 1 & \text { NtoI-1 } & 2020-04-05 \mathrm{~T} 14: 30 \mathrm{Z} & \mathrm{I} & \mathrm{A}+2 / \mathrm{P}+1 & - \\ \text { D-3 } & \# 1 & \text { A-2 } & 2020-04-05 \mathrm{~T} 14: 30 \mathrm{Z} & \mathrm{I} & \mathrm{A}+2 / \mathrm{P}+1 & - \\ \text { D-4 } & \# 1 & \text { NtoI-2 } & 2020-04-05 \mathrm{~T} 14: 30 \mathrm{Z} & \mathrm{I} & \mathrm{A}+2 / \mathrm{P}+1 & -\end{array}$

These D-records specify that the records in the RUIT slot were inserted for reasons specified in the $\boldsymbol{R}$ slot. Once more, implementors are free to develop or use any coding system they see fit for this purpose. In this case, a realism-based system for change management of ontologies and information systems is used which allows the following annotations to be made: (1) the way the absence (A) or presence (P) of a predicate $p$ compares to the existence and relevance of some PoR, (2) the beliefs of the author of $p$ in the existence and relevance of an intended PoR, and (3) the commission of mistakes of various sorts in crafting $p[44,53]$. The records $\mathrm{D} 1$ to $\mathrm{D} 4$ express by means of ' $\mathrm{A}+2$ ' that prior to the insertion of the corresponding Aand NtoI-records the absence was justified because of irrelevancy and by ' $\mathrm{P}+1$ ' that the insertion was justified and in full accordance with reality. 


\subsection{Disease-related entities and their relationships}

Less obvious than the actors on the clinician side discussed thus far is the multitude of entities on the side of the patient that must exist - or have existed - for the problem list to be a reliable summary of that part of the patient's medical history which it describes. The main question is: how far does one need to go in this analysis? Reasonable options range over a continuum from ultra-minimalist to ultramaximalist.

\subsubsection{An ultra-minimalist representation}

Ultra-minimalist in this case would be to translate the problem list in RT records that stay as closely as possible to what is expressed already while taking greater advantage of the coding systems used. Because of the existence of SNOMED CT code '55607006 | Problem (finding)', it would make sense to attempt to use SNOMED CT's concept model to express that John Doe has the stated problems at the designated times. A first step would be to assign RUIs to the problems by means of A-records and then to explicitly characterize them as such in SNOMED CT-terms using NtoC-records for the maximal temporal regions as specified in table 3, thereby assuming problems to be continuants. Although each insertion in the RTDB of records other than D-records requires the generation of a D-record, this will be omitted from here on:

\begin{tabular}{|c|c|c|c|c|c|c|c|}
\hline A\# & $\boldsymbol{R} \boldsymbol{U} \boldsymbol{I}_{\boldsymbol{a}}$ & $\boldsymbol{R} U \boldsymbol{I}_{p}$ & 'A'/ ${ }^{\prime} R$ ' & ${ }^{\prime}+S U^{\prime} /{ }^{-}-S U^{\prime}$ & \multicolumn{2}{|r|}{$T$} & \\
\hline A-10 & $\# 1$ & $\# 10$ & A & $+\mathrm{SU}$ & \multicolumn{2}{|c|}{ 2020-04-05T14:30Z } & (r23) \\
\hline A-11 & $\# 1$ & $\# 11$ & A & $+\mathrm{SU}$ & \multicolumn{2}{|c|}{ 2020-04-05T14:30Z } & $(\mathrm{r} 24)$ \\
\hline A-12 & $\# 1$ & $\# 12$ & A & $+\mathrm{SU}$ & \multicolumn{2}{|c|}{ 2020-04-05T14:30Z } & (r25) \\
\hline A-13 & $\# 1$ & $\# 13$ & A & $+\mathrm{SU}$ & \multicolumn{2}{|c|}{ 2020-04-05T14:30Z } & (r26) \\
\hline NtoC\# & $\boldsymbol{R} U \boldsymbol{I}_{p}$ & $\boldsymbol{R} U \boldsymbol{I}_{c s}: \boldsymbol{r}$ & \multicolumn{2}{|c|}{ code } & $r T$ & $t_{r}$ & \\
\hline NtoC-1 & $\# 10$ & ofClass & \multicolumn{2}{|r|}{ SCT:55607006 } & at & D1-D2273 & (r27) \\
\hline NtoC-2 & $\# 11$ & ofClass & \multicolumn{2}{|r|}{ SCT:55607006 } & at & D241-D1899 & (r28) \\
\hline NtoC-3 & $\# 12$ & ofClass & \multicolumn{2}{|r|}{ SCT:55607006 } & at & D426-D2317 & (r29) \\
\hline NtoC-4 & \#13 & ofClass & \multicolumn{2}{|r|}{ SCT:55607006 } & follows & D2317 & (r30) \\
\hline
\end{tabular}

Further specification is however required, first as concerns the periods within the maximal region during which the problems were active and inactive, respectively. This can be done using the codes ' 394774009 | Active problem (qualifier value)' and '394775005 | Inactive problem (qualifier value)' as shown for problem P3:

$\begin{array}{llllrrr}\text { NtoC\# } & \text { RUI } & \text { RUI } \text { Is: }_{\boldsymbol{r}} & \text { code } & \boldsymbol{r} \boldsymbol{T} & \boldsymbol{t} & \\ \text { NtoC-5 } & \# 12 & \text { ofClass } & \text { SCT:394774009 } & \text { at } & \text { D426-D611 } & \text { (r31) } \\ \text { NtoC-6 } & \# 12 & \text { ofClass } & \text { SCT:394775005 } & \text { at } & \text { D611-D2273 } & \text { (r32) } \\ \text { NtoC-7 } & \# 12 & \text { ofClass } & \text { SCT:394774009 } & \text { at } & \text { D2273-D2317 } & \text { (r33) }\end{array}$


This representation stays, perhaps surprisingly, within what is allowed according to SNOMED CT's concept model. Oddly, according to that model of the version used, neither an active problem nor an inactive problem is a kind of problem. Both are qualifiers which one would expect to have been introduced in SNOMED CT to qualify a problem as being active or inactive. However, the linkage concept that one would expect to be used to do so, i.e. '363713009 |Has interpretation (attribute)|' is excluded by SNOMED CT's concept model domain and range restrictions as it is designed to link concepts of type '404684003 | Clinical finding (finding)' - thus also '55607006 | Problem (finding)' - only to concepts of type '260245000 | Findings values (qualifier value)' or '263714004 |Colors (qualifier value)|' neither of which subsume the active and inactive problem qualifier values, thus once more demonstrating that SNOMED CT'S concept model, despite having exhibited major improvements over the years, is still incoherent. Declaring \#12 as being at the same time ofClass 'problem' in r25 and ofClass 'active problem' in r 31 - although 'active problem' is not a subtype of 'problem' - does not formally violate SNOMED CT's concept model as this model lacks formal disjointness statements, even where they would be needed to avoid mistakes [83].

The second further specification that is required is to qualify what sort of problem P1 to P4 - now labelled in the RTDB with RUIs \#10 to \#13 - concretely are. The same mechanism as before can be used, as here applied to P1 (\#10):

$\begin{array}{llllrrr}\text { NtoC } \# & \text { RUI } p & \boldsymbol{R U I I} \text { s: } \boldsymbol{r} & \text { code } & \boldsymbol{r} \boldsymbol{T} & \boldsymbol{t}_{\boldsymbol{r}} & \\ \text { NtoC-8 } & \# 10 & \text { ofClass } & \text { ICD9:790.29 } & \text { at } & \text { D1-D2273 } & \text { (r34) } \\ \text { NtoC-9 } & \# 10 & \text { ofClass } & \text { ICD10:R73.09 } & \text { at } & \text { D1-D2273 } & \text { (r35) } \\ \text { NtoC-10 } & \# 10 & \text { ofClass } & \text { SCT:714628002 } & \text { at } & \text { D1-D2273 } & \text { (r36) }\end{array}$

Three remarks need to be made here. One, applicable to records $r 34$ to $r 36$, is that in an actual RTDB the versions of the coding systems need to be included in the prefix of the code slot. This would lead for each problem to additional records with each new version of the coding systems. As of 2020, 'R73.09' would no longer be a valid code for \#10 since according to the 2020 version of ICD10 the new code 'R73.03' should be used. The second remark is that records r35 and r36 are veridical - at least under the assumption that the patient does indeed have the problem as argued for by the clinicians - despite the fact that the ICD10 and SNOMED CT codes were assigned later during the patient's medical history. This is because these records provide an ontological view on the underlying reality using the spectacles offered by SNOMED CT. Of course, this view could not be used prior to the development of SNOMED CT, but once developed, the view can be used for any part of reality, also in the past. The third remark to be made is that $\mathrm{r} 36$ uses the SNOMED CT code assigned as late as day 2273, and that there is no record stating that $\mathrm{P} 1$ is ofClass SCT:9414007. That is because the use of SCT:9414007 as of day 1042 is a coding error. This is another example demonstrating the difference between a posthoc analysis and a parallel one. RT allows tuples to be made about committed mistakes, but such tuples are not part of a minimalist representation. 
The final step is now to link the problems to the patient (\#7). This is done by using the relation with as defined in section 5.1 in the following NtoN-records in which the list of relata $\boldsymbol{P}$ of the NtoN-tuple type is decomposed in $\boldsymbol{R} \boldsymbol{U} \boldsymbol{I}_{\boldsymbol{s}}$ (source) and RUI (target):

$\begin{array}{llllrrr}\text { NtoN\# } & \text { RUI } & \boldsymbol{r} & \boldsymbol{R U I}_{\boldsymbol{t}} & \boldsymbol{r} \boldsymbol{T} & \boldsymbol{t}_{\boldsymbol{r}} & \\ \text { NtoN-1 } & \# 7 & \text { with } & \# 10 & \text { at } & \text { D1-D2273 } & \text { (r37) } \\ \text { NtoN-2 } & \# 7 & \text { with } & \# 11 & \text { at } & \text { D241-D1899 } & \text { (r38) } \\ \text { NtoN-3 } & \# 7 & \text { with } & \# 12 & \text { at } & \text { D426-D2317 } & \text { (r39) } \\ \text { NtoN-4 } & \# 7 & \text { with } & \# 13 & \text { follows } & \text { D2317 } & \text { (r40) }\end{array}$

\subsubsection{Minimalist representations}

Whereas an ultra-minimalist representation sugar coats a conceptual view with a realism-based interpretation, minimalist as well as all more elaborate representations need to be realism-based wherever possible. Although the problem list contained over time four problems of which at some points in time two were claimed to be simultaneously active, no clinician would argue that this problem list would indicate that this patient had at any point in time more than one condition. Note that the rather vague, yet popular, term 'condition' is used here rather than 'disease' for reasons that will be explained below. Neither is there any clinician who would argue that the patient suffered from a first disease called 'prediabetes' which then became co-morbid with a second disease called 'diabetes' but which then healed between days 426 and 611, and so forth. That would of course be nonsense. But what can be deduced through a post-hoc analysis of the problem list is that the patient's medical history evolved thus far in five phases. These five phases - not to be confused with the four 'problems' - are (1) a prediabetic one which started prior to day 1 and ended prior to day 426 , followed by (2) a diabetic phase until prior to day 611 , then again (3) a prediabetic one until prior to day 2273, followed by (4) a second diabetic phase which (5) was finally well under control, if not since day 2237 , then at least sometime later up to and including day 2317.

What would each of these phases be ontologically? Once more, ICD and SNOMED CT see it quite differently. The prediabetic phases are for ICD-9-CM phases characterized by a 'nonspecific abnormal finding', for ICD-10-CM by a 'symptom, sign or abnormal clinical and laboratory finding, not elsewhere classified', and according to SNOMED CT by a 'finding'. Parts of the $2^{\text {nd }}$ phase are annotated under P2 with codes for 'Impaired fasting glucose' which both ICD versions also see as an abnormal finding, while SNOMED CT considers it a disorder. All three systems consider the $4^{\text {th }}$ and $5^{\text {th }}$ phases to be disorders, be it, oddly, of different types.

Under OGMS, being the ontology chosen, the story is entirely different. In light of the definitions presented in section 7.1, these phases correspond to temporal parts of a disease course, a disease course being a process through which a disease is realized. An important question for an accurate ontological representation is now: 
how may disease courses, and thus diseases, has the patient exhibited over the period covered by the problem list under the view defended by OGMS? This question needs to be answered in the first place by specialists in diabetes research in collaboration with ontologists familiar with OGMS, a problem being that thus far no identity criteria have been proposed for 'disease' - neither in general, if at all possible - or through subtyping of the disease universal into categories for specific types of diseases. The view translated in RT tuples here is that this patient has only one disease, thus only one disease course, and that the phases are temporal parts of this numerically identical disease course. The alternative, still compatible with OGMS, would be that the patient suffered from a first disease (prediabetes) during phase 1 which was a predisposition to develop the $2^{\text {nd }}$ disease (diabetes) in phase 2 , that once this $2^{\text {nd }}$ disease developed, the first one ceased to exist, that then this one ceased to exist also after which the patient became sick for the second time from an instance of prediabetes, and so forth. This alternative is however quite debatable as it seems to rest on conflating disease with diagnostic criteria for disease. Along the same debatable line of thinking it could be argued that a patient with chronic pain never had 'just' chronic pain, but 'acute pain' when the pain started, then just 'pain' once the acute phase is over, while after three months - the standard diagnostic criterion for chronic pain - a third pain which would be 'chronic pain'. The more plausible analysis is that such patient had chronic pain from the very beginning, but there was no way to know that until later.

The RUIs to be generated (A-records are not shown any more from here on) are then the 5 phases (\#14 to \#18), the disease (\#19), and the disease course (\#20) which is the realization of the disease. These numerically distinct entities are introduced here because they are indirectly referenced in the problem list. Coincidentally, they satisfy also the general rule that whenever a dependent entity needs to be represented, all entities upon which it depends need to be represented as well. Both the disease course and its temporal parts, i.e. the phases, and the disease are dependent on John Doe who is already represented (\#7).

The following NtoR-records represent what the entities are instances of:

$\begin{array}{llllrrr}\text { NtoR\# } & \boldsymbol{R U} \boldsymbol{I}_{\boldsymbol{n}} & \text { inst } & \boldsymbol{R U} \boldsymbol{I}_{\boldsymbol{r}} & \boldsymbol{r} \boldsymbol{T} & \boldsymbol{t}_{\boldsymbol{r}} & \\ \text { NtoR-1 } & \# 7 & \text { BFO:instanceOf } & \text { NCIT:Homo sapiens } & \text { at } & \text { t10 } & (\mathrm{r} 41) \\ \text { NtoR-2 } & \# 14 & \text { BFO:instanceOf } & \text { BFO:process } & - & - & (\mathrm{r} 42) \\ \text { NtoR-3 } & \# 15 & \text { BFO:instanceOf } & \text { BFO:process } & - & - & (\mathrm{r} 43) \\ \text { NtoR-4 } & \# 16 & \text { BFO:instanceOf } & \text { BFO:process } & - & - & (\mathrm{r} 44) \\ \text { NtoR-5 } & \# 17 & \text { BFO:instanceOf } & \text { BFO:process } & - & - & (\mathrm{r} 45) \\ \text { NtoR-6 } & \# 18 & \text { BFO:instanceOf } & \text { BFO:process } & - & - & (\mathrm{r} 46) \\ \text { NtoR-7 } & \# 19 & \text { BFO:instanceOf } & \text { OGMS:disease } & \text { at } & \text { t11 } & (\mathrm{r} 47) \\ \text { NtoR-8 } & \# 20 & \text { BFO:instanceOf } & \text { OGMS:disease course } & - & - & (\mathrm{r} 48)\end{array}$

The minimal set of records to tie these entities together then looks as follows:

$\begin{array}{llllrr}\text { NtoN\# } & \boldsymbol{R U} \boldsymbol{I}_{\boldsymbol{s}} & \boldsymbol{R} & \boldsymbol{R U} \boldsymbol{I}_{\boldsymbol{t}} & \boldsymbol{r} \boldsymbol{T} & \boldsymbol{t}_{\boldsymbol{r}} \\ \text { NtoN-5 } & \# 19 & \text { BFO:inheresIn } & \# 7 & \text { at } & \text { t1 } 1\end{array}$




$\begin{array}{llllrrr}\text { NtoN-6 } & \# 20 & \text { BFO:realizationOf } & \# 19 & \text { at } & \text { t12 } & (\mathrm{r} 50) \\ \text { NtoN-7 } & \# 14 & \text { BFO:temporalPartOf } & \# 20 & - & - & (\mathrm{r} 51) \\ \text { NtoN-8 } & \# 15 & \text { BFO:temporalPartOf } & \# 20 & - & - & (\mathrm{r} 52) \\ \text { NtoN-9 } & \# 16 & \text { BFO:temporalPartOf } & \# 20 & - & - & (\mathrm{r} 53) \\ \text { NtoN-10 } & \# 17 & \text { BFO:temporalPartOf } & \# 20 & - & - & (\mathrm{r} 54) \\ \text { NtoN-11 } & \# 18 & \text { BFO:temporalPartOf } & \# 17 & - & - & (\mathrm{r} 55)\end{array}$

The maximal temporal region during which \#19 inheres in the patient as specified in r49 is numerically identical to the maximal temporal region during which \#19 is an instance of disease as stated in r47. This reflects two beliefs: (1) that this disease in John cannot become a disease in somebody else, and (2) that it cannot exist as anything else than as an instance of disease. The RUIs for the maximal temporal regions denoted in $\mathrm{r} 49$ and $\mathrm{r} 50$ are different. Because as argued for in section 4.2, the RUIs for temporal regions are not to be taken as being singularly unique identifiers, the use of $\mathrm{t} 11$ and $\mathrm{t} 12$ in $\mathrm{r} 49$ and $\mathrm{r} 50$ respectively leave it open whether the realization of this disease starts at the same time as the disease comes into existence. OGMS does not say anything about this in general (since there is no way of knowing in any given case). This is another example where thinking in terms of RT is helpful to identify further axioms that might have to be included in ontologies.

The precise temporal ordering of the phases can be specified by means of NtoNrecords using the appropriate relations from ISO-standard 12381:2019. The use of BFO:temporalPartOf rather than BFO:properTemporalPartOf in $\mathrm{r} 60$ reflects the fact that $\mathrm{t} 11$ and $\mathrm{t} 12$ may be singularly unique, but when they are not, t12 is shorter in duration than $\mathrm{t} 11$.

$\begin{array}{llllrrr}\text { NtoN\# } & \boldsymbol{R U} \boldsymbol{I}_{\boldsymbol{s}} & \boldsymbol{R} & \boldsymbol{R U} \boldsymbol{I}_{\boldsymbol{t}} & \boldsymbol{r} \boldsymbol{T} & \boldsymbol{t}_{\boldsymbol{r}} & \\ \text { NtoN-12 } & \# 20 & \text { co-continues } & \# 14 & - & - & (\mathrm{r} 56) \\ \text { NtoN-13 } & \# 15 & \text { Follows } & \# 14 & - & - & (\mathrm{r} 57) \\ \text { NtoN-14 } & \# 16 & \text { Follows } & \# 15 & - & - & (\mathrm{r} 58) \\ \text { NtoN-15 } & \# 17 & \text { Follows } & \# 16 & - & - & (\mathrm{r} 59) \\ \text { NtoN-16 } & \text { t12 } & \text { BFO:temporalPartOf } & \text { t11 } & - & - & \text { (r60) }\end{array}$

The nature of the various entities identified for a minimal representation has thus far only been qualified at a very high level of granularity. Clearly, record r47 does not do so at the appropriate level of granularity: it is faithful, but not adequately faithful since it does not express that \#19 is an instance of Type 2 Diabetes. To resolve this, one could start with the development of a realism-based ontology for diseases and define Type 2 Diabetes therein. Trying to achieve this through a mere mapping between the topmost concepts of SNOMED CT hierarchies, i.e. roughly the concepts which have a semantic tag different than any of the semantic tags of their immediate ancestors [26], and OGMS universals as some have proposed rather absurdly [84, Fig 5, p10], does not work, not only because of, in this case, a misinterpretation of BFO and OGMS types in the proposed mapping, but also because of 
the intrinsically heterogenous nature of the descendants of SNOMED CT subhierarchies [85]. An alternative would be to proceed as in the ultra-minimalist approach and link the disease-related entities to the relevant codes of the concept systems:

$\begin{array}{llllrrr}\text { NtoC\# } & \boldsymbol{R U I} \boldsymbol{I}_{\boldsymbol{p}} & \boldsymbol{R} \boldsymbol{U I I}_{\text {cs: }} \boldsymbol{r} & \text { code } & \boldsymbol{r} \boldsymbol{T} & \boldsymbol{t}_{\boldsymbol{r}} & \\ \text { NtoC-11 } & \# 20 & \text { ofClass } & \text { SCT: } 44054006 & \text { at } & \mathrm{t} 12 & \text { (r61) } \\ \text { NtoC-12 } & \# 14 & \text { ofClass } & \text { SCT:714628002 } & \text { at } & \mathrm{t} 13 & \text { (r62) } \\ \text { NtoC-13 } & \# 15 & \text { ofClass } & \text { SCT:313436004 } & \text { at } & \text { t14 } & \text { (r63) }\end{array}$

The absence of a satisfying realism-based account for 'problem' makes it also difficult to link the problems (\#10 to \#13) explicitly to the identified phases (\#14 tot \#18). An implicit linking can be achieved nevertheless, for instance by using NtoN records to describe the temporal relations that hold between the temporal regions during which the phases exist and the temporal regions during which the problems are stated to be active.

Finally, a minimalist representation which supports this problem list would need to account also for what is documented as problem 2 under the name 'Impaired Fasting glucose'. Although SNOMED CT classifies what is denoted by this term as a disorder in the same way as 'impaired glucose tolerance' (table 4), ICD - rather surprisingly - does a better job in characterizing these terms as denoting not a disorder in their own right, but rather signs of a disorder which are observed by performing a blood glucose test under standardized conditions of fasting or glucose administration. The results of the test would then indicate what diabetic phase the patient would be in, if in any at all. This requires minimally two other entities to be referenced (with of course the generation of the required corresponding core records), namely the portion of blood drawn to perform the test, and the concentration of glucose in that portion of blood. How to do this using records of the types discussed thus far should now be clear.

\subsubsection{Maximal and ultra-maximal representations}

A maximal representation in the context of this problem list would require all entities to be represented that are referenced directly or indirectly in any data element of that part of the EMR system's back-end database that is described in table 3. It would further require those entities on the side of the patient that must exist or have existed for any of the former entities to exist or to have come into existence to be listed wherever they are relevant for the patient's care. And finally, if any of the entities are dependent, the independent ones upon which they depend need to be brought into the picture as well. Examples would be the blood draw which created the sample, who performed the blood draw, the test procedure used, and so forth. Although all these entities exist(ed), few of them would be singularly uniquely identifiable in a post-hoc scenario, while otherwise provisions could be taken to ensure that information is reported. Once this is done, NtoN-records can be used to describe the various relationships, for instance between the clinicians identified in section 
7.3 and the assertions they made. Also included in a maximalist representation would be the use of F-tuples expressing the beliefs in the faithfulness of the various records generated and how they correspond with the beliefs that the clinicians must have held in order to make the annotations in the record.

The ultra-maximalist representation would further include RT-representations of the relevant parts of the back-end database of the EMR system itself [59]. This would then make it possible to represent in the RTS, for example, the change (and explanation thereof) in SNOMED CT code for problem P1 on day 2273, before that day being '9414007 - Impaired glucose tolerance (disorder)' and then '714628002 - Prediabetes (finding)'. Addressing these representations is however beyond the scope of this chapter.

\section{Conclusion and further reading}

The idea of Referent Tracking was first introduced in 2005 as a new paradigm for entry and retrieval of data in the EMR [5] thereby building further on what became the foundations for the Relation Ontology [22]. The paper contains an easy to read introduction to the sort of problems that arise when general terms taken from terminologies or ontologies (such as 'fracture' and 'left femur') are used in the EMR to refer to particular entities on the side of a patient such as the particular fracture that John suffered from in 2005 in his left femur. It explains how all ambiguities can be avoided by referring to such entities by means of identifiers instead of, or, better, in addition to, codes. A more thorough and formal discussion of the types of statements that are required as well as the technical infrastructure for real implementations followed shortly thereafter [4].

Of course, RT presents some challenges of its own. One specific problem is how to represent phenomena commonly expressed by statements such as: "no history of diabetes', 'hypertension ruled out', 'absence of metastases in the lung', and 'abortion was prevented'. Such statements seem at first sight to present a problem for $\mathrm{RT}$, since there are here no entities on the side of the patient to which unique identifiers can be attached. For concept-based systems, this is not an issue since entities such as 'diabetes' or 'no diabetes' are both happily classified as 'concepts', no further questions being asked. This problem was solved on the realist side by introducing the 'lacks' relation which holds between particulars and universals, thereby remaining faithful to the principles of unqualified realism within an EMR regime based on the idea of faithfulness to clinical reality $[10,86]$.

Another challenge is keeping track of the different kinds of changes, reflecting for example: (1) changes in the underlying reality, either in a specific patient's condition or the world in general; (2) changes in our understanding; (3) reassessments of what is considered to be relevant for inclusion in a referent tracking database, or (4) encoding mistakes introduced during data entry. These issues could be addressed from the perspective of versioning in ontologies and repositories [53]. The method developed was afterwards used to assess the history mechanism of SNOMED CT 
[29], forecasting the quality of future versions of the Gene Ontology [87], and analyzing the adequateness of ontology mapping efforts [88].

Many papers describe the potential benefits of the use of RT in a variety of contexts and purposes. Examples are applications for the Semantic Web [89] and the Internet of Things [90], adverse event reporting [11, 91], expanding the representational power of i2b2 [13], properly structuring medical diagnoses when using ICD codes [92] thereby indicating what they are precisely about [93] and the extent to which they are warranted [94]. RT has been shown to be useful in integrating data from different sources [59] which may contain multiple references to the same entity, and incomplete or conflicting information thus requiring tracking of the provenance of information when making decisions on what is the actual phenotype of a person [95]. This includes applications outside the domain of healthcare such as enterprise information systems [96], homeland security [9] and the validation of Joint Battle Management Language messages [97].

List of abbreviations

\begin{tabular}{|l|l|}
\hline BFO & Basic Formal Ontology \\
\hline EAV & Entity - Attribute - Value \\
\hline EMR & Electronic Medical Record \\
\hline EAV & Entity - Attribute - Value \\
\hline IAO & Information Artifact Ontology \\
\hline ICD & International Classification of Diseases \\
\hline IUI & Instance Unique Identifier \\
\hline OBI & Ontology for Biomedical Investigations \\
\hline OGMS & Ontology for General Medical Science \\
\hline PoR & Portion of Reality \\
\hline RDB & Relational Database \\
\hline RO & Relation Ontology \\
\hline RT & Referent Tracking \\
\hline RTDB & Referent Tracking Database \\
\hline RTS & Referent Tracking System \\
\hline RU & Representational Unit \\
\hline RUI & Referent Unique Identifier \\
\hline SCTID & SNOMED CR Identifier \\
\hline
\end{tabular}

\section{Acknowledgments}

We are grateful to Christian Stoeckert and Barry Smith for a careful reading of this chapter and many suggestions for making it easier to understand. Part of the research reported in this publication was supported by the National Center for Advancing Translational Sciences of the National Institutes of Health under award 
number KL2TR001413 to the University at Buffalo. The content is solely the responsibility of the authors and does not necessarily represent the official views of the NIH.

\section{References}

[1] Rosenbloom ST, Miller RA, Johnson KB, Elkin PL, Brown SH. Interface terminologies: facilitating direct entry of clinical data into electronic health record systems. J Am Med Inform Assoc. 2006 May-Jun;13(3):277-88.

[2] Schulz S, Martínez-Costa C, Karlsson D, Cornet R, Brochhausen M, Rector A. An Ontological Analysis of Reference in Health Record Statements. In: Pawel Garbacz OK, editor. The 8th International Conference on Formal Ontology in Information Systems, Rio de Janeiro, Brazil, September 22-25, 2014; 2014; Amsterdam: IOS Press; 2014. p. 289-302.

[3] Smith B, Ceusters W. An ontology-based methodology for the migration of biomedical terminologies to electronic health records. AMIA Annu Symp Proc. 2005:704-8.

[4] Ceusters W, Smith B. Strategies for referent tracking in electronic health records. J Biomed Inform. 2006 Jun;39(3):362-78.

[5] Ceusters W, Smith B. Tracking referents in electronic health records. Stud Health Technol Inform. 2005;116:71-6.

[6] Rudnicki R, Ceusters W, Manzoor S, Smith B. What particulars are referred to in Electronic Health Record data? A case study in integrating Referent Tracking into an EHR application. AMIA Annu Symp Proc. 2007:630-4.

[7] Manzoor S, Ceusters W, Rudnicki R. Implementation of a Referent Tracking System. International Journal of Healthcare Information Systems and Informatics. 2007;2(4):41-58.

[8] Manzoor S, Ceusters W, Rudnicki R. A Middleware Approach to Integrate Referent Tracking in EHR Systems. In: Teich JM, Suermondt J, C H, editors. Proceedings of the American Medical Informatics Association 2007 Annual Symposium Biomedical and Health Informatics: From Foundations to Applications to Policy. Chicago IL2007. p. 503-7.

[9] Ceusters W, Manzoor S. How to track Absolutely Everything? In: Obrst L, Janssen T, Ceusters W, editors. Ontologies and Semantic Technologies for the Intelligence Community Frontiers in Artificial Intelligence and Applications. Amsterdam: IOS Press; 2010. p. 13-36.

[10] Ceusters W, Elkin P, Smith B. Negative findings in electronic health records and biomedical ontologies: a realist approach. Int J Med Inform. 2007 Dec;76 Suppl 3:S326-33. 
[11] Ceusters W, Capolupo M, de Moor G, Devlies J, Smith B. An evolutionary approach to realism-based adverse event representations. Methods Inf Med. 2011;50(1):62-73.

[12] Ceusters W, Blaisure J. Caveats for the Use of the Active Problem List as Ground Truth for Decision Support. Stud Health Technol Inform. 2018;255:10-4.

[13] Blaisure JC, Ceusters WM. Enhancing the Representational Power of i2b2 through Referent Tracking. AMIA Annu Symp Proc. 2018;2018:262-71.

[14] Ceusters W, Blaisure J. A Realism-Based View on Counts in OMOP's Common Data Model. Stud Health Technol Inform. 2017;237:55-62.

[15] Blaisure JC, Ceusters WM. Improving the 'Fitness for Purpose' of Common Data Models through Realism Based Ontology. AMIA Annu Symp Proc. 2017;2017:440-7.

[16] Blaisure J, Ceusters W. Business Rules to Improve Secondary Data Use of Electronic Healthcare Systems. Stud Health Technol Inform. 2017;235:303-7.

[17] Schuler JC, Ceusters WM. The Problems of Realism-Based Ontology Design: a Case Study in Creating Definitions for an Application Ontology for Diabetes Camps. AMIA Annu Symp Proc. 2017;2017:1517-26.

[18] Ceusters W, Smith B. Biomarkers in the ontology for general medical science. Stud Health Technol Inform. 2015;210:155-9.

[19] Smith B, Ceusters W. Ontological realism: A methodology for coordinated evolution of scientific ontologies. Appl Ontol. 2010;5(3-4):139-88.

[20] Arp R, Smith B, Spear AD. Building ontologies with basic formal ontology. Cambridge, Massachusetts: The MIT Press,; 2015.

[21] Smith B, Ceusters W. Aboutness: Towards Foundations for the Information Artifact Ontology. International Conference on Biomedical Ontology; July 27-30; Lisbon, Portugal2015. p. 47-51.

[22] Smith B, Ceusters W, Klagges B, Kohler J, Kumar A, Lomax J, et al. Relations in biomedical ontologies. Genome Biology. 2005 2005;6(5).

[23] Scheuermann RH, Ceusters W, Smith B. Toward an ontological treatment of disease and diagnosis. Summit Transl Bioinform. 2009 Mar 1;2009:116-20.

[24] Bandrowski A, Brinkman R, Brochhausen M, Brush MH, Bug B, Chibucos MC, et al. The Ontology for Biomedical Investigations. PLoS One. 2016;11(4):e0154556.

[25] Ceusters W, Mullin S. Expanding Evolutionary Terminology Auditing with Historic Formal and Linguistic Intensions: A Case Study in SNOMED CT. Stud Health Technol Inform. 2019 Aug 21;264:65-9.

[26] Bona JP, Ceusters W. Mismatches between major subhierarchies and semantic tags in SNOMED CT. J Biomed Inform. 2018 May;81:1-15.

[27] Ceusters W, Bona JP. Analyzing SNOMED CT's Historical Data: Pitfalls and Possibilities. AMIA Annu Symp Proc. 2016;2016:361-70.

[28] Ceusters W. Applying Evolutionary Terminology Auditing to SNOMED CT. AMIA Annu Symp Proc. 2010 Nov 13;2010:96-100. 
[29] Ceusters WM, Spackman KA, Smith B. Would SNOMED CT benefit from realism-based ontology evolution? AMIA Annu Symp Proc. 2007 Oct 11:105-9.

[30] Ceusters W, Smith B, Kumar A, Dhaen C. Ontology-based error detection in SNOMED-CT. Stud Health Technol Inform. 2004;107(Pt 1):482-6.

[31] Ceusters W, Michelotti A, Raphael KG, Durham J, Ohrbach R. Perspectives on next steps in classification of oro-facial pain - part 1: role of ontology. J Oral Rehabil. 2015 Dec;42(12):926-41.

[32] Nixdorf DR, Drangsholt MT, Ettlin DA, Gaul C, De Leeuw R, Svensson $\mathrm{P}$, et al. Classifying orofacial pains: a new proposal of taxonomy based on ontology. J Oral Rehabil. 2012 Mar;39(3):161-9.

[33] Ceusters W, Jensen M, Diehl AD. Ontological Realism for the Research Domain Criteria for Mental Disorders. Stud Health Technol Inform. 2017;235:431-5.

[34] Ceusters W, Smith B. On Defining Bruxism. Stud Health Technol Inform. 2018;247:551-5.

[35] Doing-Harris K, Meystre SM, Samore M, Ceusters W. Applying ontological realism to medically unexplained syndromes. Stud Health Technol Inform. 2013;192:97-101.

[36] Grewe N, Jansen L, Smith B. Permanent generic relatedness and silent change. Formal Ontology and Information Systems: CEUR, Vol. 1060; 2016. p. 1-5.

[37] Vogt L, Grobe P, Quast B, Bartolomaeus T. Top-level categories of constitutively organized material entities--suggestions for a formal toplevel ontology. PLoS One. 2011 Apr 21;6(4):e18794.

[38] Vogt L, Grobe P, Quast B, Bartolomaeus T. Accommodating ontologies to biological reality--top-level categories of cumulative-constitutively organized material entities. PLoS One. 2012;7(1):e30004.

[39] Vallicella WF. Three Conceptions of States of Affairs. Noûs. 2000;34(2):237-59.

[40] Smith B. Against Fantology. In: Reicher ME, Marek JC, editors. Experience and Analysis. Wien2005. p. 153-70.

[41] Yu AC, Cimino JJ. A comparison of two methods for retrieving ICD-9CM data: the effect of using an ontology-based method for handling terminology changes. J Biomed Inform. 2011 Apr;44(2):289-98.

[42] Wang S, Schlobach S, Klein M. Concept drift and how to identify it. J Web Semant. 2011 2011/09/01/;9(3):247-65.

[43] Ceusters W. SNOMED CT's RF2: Is the future bright? Stud Health Technol Inform. 2011;169:829-33.

[44] Seppälä S, Smith B, Ceusters W. Applying the Realism-Based OntologyVersioning Method for Tracking Changes in the Basic Formal Ontology. In: Garbacz P, Kutz O, editors. Formal Ontology in Information Systems (FOIS 2014): IOS Press; 2014. p. 227-40. 
[45] Smith B, Kusnierczyk W, Schober D, Ceusters W, editors. Towards a reference terminology for ontology research and development in the biomedical domain. CEUR Workshop Proceedings; 20062006.

[46] Ceusters W, Steurs F, Zanstra P, Van Der Haring E, Rogers J. From a time standard for medical informatics to a controlled language for health. Int $\mathrm{J}$ Med Inform. 1998 Feb;48(1-3):85-101.

[47] Ceusters W, Buekens F, DeMoor G, Bernauer J, DeKeyser L, Surjan G. TSMI: a CEN/TC251 standard for time specific problems in healthcare informatics and telematics. Int J Med Inform. 1997 Sep;46(2):87-101.

[48] Haslam N. Kinds of kinds: A conceptual taxonomy of psychiatric categories. Philosophy, Psychiatry, and Psychology. 2002;9(3):203-17.

[49] Nadkarni PM, Brandt C. Data extraction and ad hoc query of an entityattribute-value database. J Am Med Inform Assoc. 1998 NovDec;5(6):511-27.

[50] Nadkarni PM, Marenco L, Chen R, Skoufos E, Shepherd G, Miller P. Organization of heterogeneous scientific data using the EAV/CR representation. J Am Med Inform Assoc. 1999 Nov-Dec;6(6):478-93.

[51] Kumar SK, Harding JA. Ontology mapping using description logic and bridging axioms. Computers in industry. 2013;64(1):19-28.

[52] Ceusters W. Dealing with Mistakes in a Referent Tracking System. In: Hornsby K, editor. Proceedings of Ontology for the Intelligence Community 2007 (OIC-2007). Columbia MA2007. p. 5-8.

[53] Ceusters W, Smith B. A realism-based approach to the evolution of biomedical ontologies. Annual Symposium proceedings AMIA Symposium. 2006:121-5.

[54] Ceusters W. Dealing with mistakes in a referent tracking system. Ontology for the Intelligence Community (OIC-2007); Columbia, Maryland2007. p. $5-8$.

[55] Chen C, Hanna J, Talburt JR, Brochhausen M, Hogan WR. A Demonstration of Entity Identity Information Management Applied to Demographic Data in a Referent Tracking System. CEUR Workshop Proceedings. 2013;1060:136-7.

[56] Weiskopf NG, Bakken S, Hripcsak G, Weng C. A Data Quality Assessment Guideline for Electronic Health Record Data Reuse. EGEMS (Wash DC). 2017 Sep 4;5(1):14.

[57] Bittner T, Smith B. A Theory of Granular Partitions. In: Duckham M, Goodchild MF, Worboy MF, editors. Foundations of Geographic Information Science. London: Taylor \& Francis Books; 2003. p. 117-51.

[58] Hill DP, Smith B, McAndrews-Hill MS, Blake JA. Gene Ontology annotations: what they mean and where they come from. BMC Bioinformatics. 2008 Apr 29;9 Suppl 5:S2.

[59] Ceusters W, Hsu CY, Smith B, editors. Clinical data wrangling using ontological realism and referent tracking. CEUR Workshop Proceedings; 20142014. 
[60] Ceusters W, Hogan WR, editors. An ontological analysis of diagnostic assertions in electronic healthcare records. Conference on Biomedical Ontology; 2015 July 27-30; Lisbon, Portugal.

[61] Wright A, McCoy AB, Hickman TT, Hilaire DS, Borbolla D, Bowes WA, 3rd, et al. Problem list completeness in electronic health records: A multisite study and assessment of success factors. Int J Med Inform. 2015 Oct;84(10):784-90.

[62] Poissant L, Taylor L, Huang A, Tamblyn R. Assessing the accuracy of an inter-institutional automated patient-specific health problem list. BMC Med Inform Decis Mak. 2010 Feb 23;10:10.

[63] Weed L. Medical records that guide and teach. New England Journal of Medicine. 1968;278:593-600.

[64] Hodge CM, Narus SP. Electronic problem lists: a thematic analysis of a systematic literature review to identify aspects critical to success. J Am Med Inform Assoc. 2018 Mar 14.

[65] Tabak AG, Herder C, Rathmann W, Brunner EJ, Kivimaki M. Prediabetes: a high-risk state for diabetes development. Lancet. 2012 Jun 16;379(9833):2279-90.

[66] Porter AS, O'Callaghan J, Englund KA, Lorenz RR, Kodish E. Problems with the problem list: challenges of transparency in an era of patient curation. J Am Med Inform Assoc. 2020 Jun 1;27(6):981-4.

[67] Sockolow PS, Le NB, Yang Y, Potashnik S, Bass EJ, Bowles KH. Incongruence of Patient Problem Information Across Three Phases of Home Care Admission: There's a Problem with the Problem List. Stud Health Technol Inform. 2019 Aug 21;264:803-7.

[68] Rajbhandari P, Auron M, Worley S, Marks M. Improving Documentation of Inpatient Problem List in Electronic Health Record: A Quality Improvement Project. J Patient Saf. 2018 Apr 19.

[69] Krauss JC, Boonstra PS, Vantsevich AV, Friedman CP. Is the problem list in the eye of the beholder? An exploration of consistency across physicians. J Am Med Inform Assoc. 2016 Sep;23(5):859-65.

[70] Bansal N. Prediabetes diagnosis and treatment: A review. World J Diabetes. 2015 Mar 15;6(2):296-303.

[71] Taylor R, Barnes AC. Can type 2 diabetes be reversed and how can this best be achieved? James Lind Alliance research priority number one. Diabetic Medicine. 2019;36(3):308-15.

[72] American Diabetes A. Diagnosis and classification of diabetes mellitus. Diabetes Care. 2014 Jan;37 Suppl 1:S81-90.

[73] American Diabetes A. 2. Classification and Diagnosis of Diabetes: Standards of Medical Care in Diabetes-2020. Diabetes Care. 2020 Jan;43(Suppl 1):S14-S31.

[74] Elliott S. Research Problems. The British Journal for the Philosophy of Science. 2019. 
[75] Agrawal A, He Z, Perl Y, Wei D, Halper M, Elhanan G, et al. The readiness of SNOMED problem list concepts for meaningful use of electronic health records. Artif Intell Med. 2013 Jun;58(2):73-80.

[76] Smith B, Ceusters W. HL7 RIM: an incoherent standard. Stud Health Technol Inform. 2006;124:133-8.

[77] Smith B, Vizenor L, Ceusters W. Human Action in the Healthcare Domain: A Critical Analysis of HL7's Reference Information Model. . In: Svennerlind C, Almäng J, Ingthorsson R, editors. Johanssonian Investigations: Essays in Honour of Ingvar Johansson on His Seventieth Birthday. Frankfurt: Ontos Verlag; 2013. p. 554-73.

[78] Health Level 7. FHIR Release 4 (Technical Correction \#1) (v4.0.1) Resource Condition. 2019 [updated Nov 1, 2019]; Available from: http://hl7.org/fhir/condition.html.

[79] Smith B, Ashburner M, Rosse C, Bard J, Bug W, Ceusters W, et al. The OBO Foundry: coordinated evolution of ontologies to support biomedical data integration. Nat Biotechnol. 2007 Nov;25(11):1251-5.

[80] Schriml LM, Mitraka E. The Disease Ontology: fostering interoperability between biological and clinical human disease-related data. Mammalian genome : official journal of the International Mammalian Genome Society. 2015 Oct;26(9-10):584-9.

[81] Shefchek KA, Harris NL, Gargano M, Matentzoglu N, Unni D, Brush M, et al. The Monarch Initiative in 2019: an integrative data and analytic platform connecting phenotypes to genotypes across species. Nucleic Acids Res. 2020 Jan 8;48(D1):D704-D15.

[82] Cappelen H, Lepore E, McKeever M. Quotation. In: Zalta EN, editor. The Stanford Encyclopedia of Philosophy (Summer 2020 Edition) 2020.

[83] Wang Y, Halper M, Wei D, Gu H, Perl Y, Xu J, et al. Auditing complex concepts of SNOMED using a refined hierarchical abstraction network. J Biomed Inform. $2012 \mathrm{Feb} ; 45(1): 1-14$.

[84] El-Sappagh S, Franda F, Ali F, Kwak KS. SNOMED CT standard ontology based on the ontology for general medical science. BMC Med Inform Decis Mak. 2018 Aug 31;18(1):76.

[85] Lopez-Garcia P, Schulz S. Structural Patterns under X-Rays: Is SNOMED CT Growing Straight? PLoS One. 2016;11(11):e0165619.

[86] Ceusters W, Elkin P, Smith B. Referent tracking: the problem of negative findings. Stud Health Technol Inform. 2006;124:741-6.

[87] Ceusters W. Applying evolutionary terminology auditing to the Gene Ontology. J Biomed Inform. 2009 Jun;42(3):518-29.

[88] Ceusters W. Towards A Realism-Based Metric for Quality Assurance in Ontology Matching. In: Bennett B, Fellbaum C, editors. Fr Art Int. Amsterdam: IOS Press; 2006. p. 321-32.

[89] Ceusters W, Smith B. Referent tracking for treatment optimisation in schizophrenic patients: A case study in applying philosophical ontology to diagnostic algorithms. Web semantics. 2006 Sep 1;4(3):229-36. 
[90] Ceusters W, Bona J. Ontological Foundations for Tracking Data Quality through the Internet of Things. Stud Health Technol Inform. 2016;221:748.

[91] Ceusters W, Capolupo M, Smith B, De Moor G. An evolutionary approach to the representation of adverse events. Stud Health Technol Inform. 2009;150:537-41.

[92] Hogan WR, Ceusters W. Diagnosis, misdiagnosis, lucky guess, hearsay, and more: an ontological analysis. J Biomed Semantics. 2016 Sep 15;7(1):54.

[93] Hogan W. Representing the Aboutness of a Diagnosis. Stud Health Technol Inform. 2018;247:805-9.

[94] Limbaugh D, Kasmier D, Ceusters W, Smith B. Warranted Diagnosis. Proceedings of the International Conference on Biomedical Ontology (ICBO), Buffalo, NY2019. p. 1-10.

[95] Stoeckert CJ, Birtwell DL, Freedman H, Miller MA, Williams H. Transforming and Unifying Research with Biomedical Ontologies: The Penn TURBO Project. ICBO 2018; August 7-10, 2018; Corvallis, OR: CEUR Workshop Proceedings; 2018.

[96] Ceusters W, Smith B. Referent Tracking for Corporate Memories. In: Rittgen P, editor. Handbook of Ontologies for Business Interaction. New York and London: Idea Group Publishing; 2007. p. 34-46.

[97] Manzoor S, Ceusters W, Smith B, editors. Referent tracking for command and control messaging systems. CEUR Workshop Proceedings; 2009 2009. 